\title{
The limit of small Rossby numbers for randomly forced quasi-geostrophic equation on $\beta$-plane
}

\author{
Sergei Kuksin*, Alberto Maiocchi ${ }^{\S}$
}

\begin{abstract}
We consider the $2 \mathrm{~d}$ quasigeostrophic equation on the $\beta$-plane for the stream function $\psi$, with dissipation and a random force:

$(*) \quad(-\Delta+K) \psi_{t}-\rho J(\psi, \Delta \psi)-\beta \psi_{x}=\langle$ random force $\rangle-\kappa \Delta^{2} \psi+\Delta \psi$.

Here $\psi=\psi(t, x, y), x \in \mathbb{R} / 2 \pi L \mathbb{Z}, y \in \mathbb{R} / 2 \pi \mathbb{Z}$. For typical values of the horizontal period $L$ we prove that the law of the action-vector of a solution for $(*)$ (formed by the halves of the squared norms of its complex Fourier coefficients) converges, as $\beta \rightarrow \infty$, to the law of an action-vector for solution of an auxiliary effective equation, and the stationary distribution of the action-vector for solutions of $(*)$ converges to that of the effective equation. Moreover, this convergence is uniform in $\kappa \in(0,1]$. The effective equation is an infinite system of stochastic equations which splits into invariant subsystems of complex dimension $\leq 3$; each of these subsystems is an integrable hamiltonian system, coupled with a Langevin thermostat. Under the iterated limits $\lim _{L=\rho \rightarrow \infty} \lim _{\beta \rightarrow \infty}$ and $\lim _{\kappa \rightarrow 0} \lim _{\beta \rightarrow \infty}$ we get similar systems. In particular, none of the three limiting systems exhibits the energy cascade to high frequencies.
\end{abstract}

\section{Introduction}

\subsection{Equations}

The quasi-geostrophic equation on the $\beta$-plane for the stream-function $\psi(\mathbf{x})$ describes the horizontal motion of the atmosphere and the ocean. In the case of atmosphere, $\psi$ is defined on a strip of the two-dimensional sphere around a mid-latitude parallel, while in the case of oceans it is usually defined on a bounded domain of $\mathbb{R}^{2}$. We will consider the equation in the strip, under the double-periodic boundary condition

$$
\mathbf{x}=(x, y) \in \mathbb{T}_{L, 1}^{2}=\mathbb{R} /(2 \pi L \mathbb{Z}) \times S^{1}, \quad S^{1}=\mathbb{R} / 2 \pi \mathbb{Z},
$$

where $L$ is the ratio of the horizontal to the vertical scale. Usually it is assumed to be large, $L \gg 1$. On the contrary the vertical scale (we normalised it to one) is relatively short, so the Coriolis force has approximately a linear dependence on $y$, and the equation takes the form

$$
(-\Delta+K) \psi_{t}(t, \mathbf{x})-\rho J(\psi, \Delta \psi)-\beta \psi_{x}=0, \quad \mathbf{x}=(x, y) \in \mathbb{T}_{L, 1}^{2}
$$

* CNRS and I.M.J, Université Paris Diderot-Paris 7, Paris, France, e-mail: kuksin@math.jussieu.fr

$\S$ Laboratoire de Mathématiques, Université de Cergy-Pontoise, 2 avenue Adolphe Chauvin, Cergy-Pontoise, France. 
Here $K \geq 0$ is the Froude number, $J(\psi, \Delta \psi)$ denotes the Jacobian determinant of the vector $(\psi, \Delta \psi),{ }^{1} \beta$ is a constant parameter controlling the gradient of the Coriolis force and $\rho$ is the scale of the "physical" stream function (accordingly, we intend to study solutions $\psi$ of eq. (0.1) of order one). ${ }^{2}$ The same equation describes the drift waves in plasma and is usually called in the plasma physics the Hasegawa-Mima equation (see [CFT13] for a recent study of a simplified version of this equation in 3d). With minimal changes, our approach applies to eq. (0.1) with the Dirichlet boundary conditions on the strip's boundary:

$$
(x, y) \in Q:=\mathbb{R} /(2 \pi L \mathbb{Z}) \times[0, \pi],\left.\quad \psi(t, x, y)\right|_{[0, \infty) \times \partial Q}=0 .
$$

In this case we should argue as below, decomposing solutions $\psi$ not in the exponential basis on $\mathbb{T}_{L, 1}^{2}$, but in the basis, formed by the functions $e^{i L^{-1} k_{x} x} \sin \left(k_{y} y\right), k_{x} \in \mathbb{Z}, k_{y} \in \mathbb{N}$.

In order to take into account the kinematic viscosity of the atmosphere, its friction against the surface (called the Ekman damping) as well as various external influences, the equation is modified by dissipation and random force (see [Ped79], Chap. 4, and [BDW98]):

$$
\begin{aligned}
(-\Delta+K) \psi_{t}(t, \mathbf{x})-\rho J(\psi, \Delta \psi)-\beta \psi_{x} & =\frac{d}{d t} \sum_{\mathbf{k} \in \mathbb{Z}_{*}^{2}} d_{\mathbf{k}} \boldsymbol{\beta}^{\mathbf{k}}(t) e^{i \mathbf{k}_{L} \cdot \mathbf{x}} \\
-\kappa \Delta^{2} \psi+\Delta \psi, \quad \psi & =\psi(t, \mathbf{x}), \quad \mathbf{x} \in \mathbb{T}_{L, 1}^{2} .
\end{aligned}
$$

Here the term $\Delta \psi$ represents the properly scaled Ekman damping and $-\kappa \Delta^{2} \psi, 0<\kappa \leq 1$, is the kinematic viscosity; for the Earth atmosphere $\kappa$ is very small (but positive). For $\mathbf{k}=$ $\left(k_{x}, k_{y}\right) \in \mathbb{Z}_{*}^{2}=\mathbb{Z}^{2} \backslash\{0\}, \mathbf{k}_{L}$ denotes the vector ${ }^{3}$

$$
\mathbf{k}_{L}=\left(k_{x} / L, k_{y}\right) .
$$

The numbers $d_{\mathbf{k}}$ are real non-zero and even in $\mathbf{k}$, i.e.

$$
d_{\mathbf{k}}=d_{-\mathbf{k}} \neq 0 \quad \forall \mathbf{k} .
$$

They decay with $|\mathbf{k}|$ in such a way that $B_{2}<\infty$, where for $r \geq 0$

$$
B_{r}:=2 \sum_{\mathbf{k} \in \mathbb{Z}_{*}^{2}}\left|\mathbf{k}_{L}\right|^{2 r}\left|b_{\mathbf{k}}\right|^{2} \leq \infty
$$

The processes $\boldsymbol{\beta}^{\mathbf{k}}(t), \mathbf{k} \in \mathbb{Z}_{*}^{2}$, are standard complex Wiener processes. That is $\boldsymbol{\beta}^{\mathbf{k}}(t)=W_{+}^{\mathbf{k}}(t)+$ $i W_{-}^{\mathbf{k}}(t)$, where $W_{+}^{\mathbf{k}}$ and $W_{-}^{\mathbf{k}}$ are standard independent real Wiener processes. The process $\boldsymbol{\beta}^{\mathbf{k}}$ and $\boldsymbol{\beta}^{\mathbf{j}}$ are independent if $\mathbf{k} \neq \pm \mathbf{j}$, with the reality constraint $\boldsymbol{\beta}^{-\mathbf{k}}=\overline{\boldsymbol{\beta}}^{\mathbf{k}}$ for all $\mathbf{k}$. Abusing language we will say that the processes $\boldsymbol{\beta}^{\mathbf{k}}(t), \mathbf{k} \in \mathbb{Z}_{*}^{2}$, are standard independent complex

\footnotetext{
${ }^{1}$ That is, $J(u(\mathbf{x}), v(\mathbf{x}))=u_{x} v_{y}-u_{y} v_{x}$.

${ }^{2}$ The velocity field of the flow is $u=\left(-\psi_{y}, \psi_{x}\right)^{t}$. So the vorticity is $\omega=$ curl $u=-\Delta \psi$, the Jacobian determinant is $J(\psi, \Delta \psi)=-(u \cdot \nabla) \omega$, and eq. (0.1) with $K=0$ may be written as $\omega_{t}+\rho(u \cdot \nabla) \omega-\beta u^{2}=0$. This is the (scaled) $2 \mathrm{~d}$ Euler equation, perturbed by Coriolis term $-\beta u^{2}$. Accordingly, if $K=0$, then eq. $(0.2)$ below, which we study in this work, is the stochastic 2d Navier-Stokes equation, perturbed by the Coriolos force and the Ekman damping.

${ }^{3}$ In the following, the notation $\mathbf{k}_{L}$ will be often used alongside that which explicitly involves $L$ in order to abbreviate formulas.
} 
Wiener processes with the reality constraint. The random force which stirs eq. (0.2) is a nondegenerate and sufficiently smooth in $\mathbf{x}$ real-valued random function. Last years stochastically forced $\beta$-plane equations have got much attention in the meteorological literature, e.g. see the papers [FMVE05, DG04] and the numerous works which quote them. Eq. (0.2) with $\beta=0$ is traditionally used in physics as a model for the $2 \mathrm{~d}$ turbulence, e.g. see in [Ber00].

The space-mean of solution $\psi$ is a time-independent quantity which is assumed to vanish:

$$
\langle\psi\rangle(t):=\int_{\mathbb{T}_{L, 1}^{2}} \psi(t, \mathbf{x}) d \mathbf{x} \equiv 0 .
$$

If we write $\psi(t, \mathbf{x})$ as a Fourier series, $\psi(t, \mathbf{x})=\sum_{\mathbf{k} \in \mathbb{Z}_{*}^{2}} v_{\mathbf{k}} e^{i \mathbf{k}_{L} \cdot \mathbf{x}}$, where $v_{\mathbf{k}}$ are complex numbers such that

$$
v_{-\mathbf{k}}=\bar{v}_{\mathbf{k}},
$$

then, denoting

$$
\gamma_{\mathbf{k}}=\left(\kappa\left|\mathbf{k}_{L}\right|^{4}+\left|\mathbf{k}_{L}\right|^{2}\right) /\left(K+\left|\mathbf{k}_{L}\right|^{2}\right), \quad b_{\mathbf{k}}=d_{\mathbf{k}} /\left(K+\left|\mathbf{k}_{L}\right|^{2}\right),
$$

we rewrite $(0.2)$ as the system

$$
\begin{aligned}
\dot{v}_{\mathbf{k}}-i \beta \frac{k_{x}}{L\left(K+\left|\mathbf{k}_{L}\right|^{2}\right)} v_{\mathbf{k}}= & \frac{\rho}{L\left(K+\left|\mathbf{k}_{L}\right|^{2}\right)} \sum_{\mathbf{j}, \mathbf{n} \in \mathbb{Z}_{*}^{2}}\left|\mathbf{n}_{L}\right|^{2}(\mathbf{j} \times \mathbf{n}) v_{\mathbf{j}} v_{\mathbf{n}} \delta_{\mathbf{k}}^{\mathbf{j n}} \\
& -\gamma_{\mathbf{k}} v_{\mathbf{k}}+b_{\mathbf{k}} \dot{\boldsymbol{\beta}}^{\mathbf{k}}(t), \quad \mathbf{k} \in \mathbb{Z}_{*}^{2} .
\end{aligned}
$$

Here $\mathbf{j} \times \mathbf{n}=j_{x} n_{y}-j_{y} n_{x}$, and we use the standard in physics notation (see [Naz11])

$$
\delta_{\mathbf{k}}^{\mathbf{j n}}=\left\{\begin{array}{cc}
1 & \text { if } \mathbf{j}+\mathbf{n}-\mathbf{k}=0 \\
0 & \text { otherwise }
\end{array} .\right.
$$

The Rossby number of a solution $\psi$ of (0.2) of order one is Ro $:=\frac{\rho}{\beta L}$, and we are interested in the behaviour of solutions $\psi$ under the limit of small Ro. This limit is relevant for meteorology and climatology, see [Ped79]. Indeed, as it was first pointed out by Rossby [ $\left.\mathrm{R}^{+} 39\right]$, under this limit the characteristic features of large scale solutions of the 3d Navier Stokes equation (3d NSE) of a sheet of fluid on a rotating sphere can be well approximated, at least in mid latitudes, by solutions of (0.2). Among various linear modes of oscillations in the linearized inviscid 3d NSE for rotating fluid, which are called Poincaré, Kelvin and Rossby modes (see [GSR06] for more details), only the Rossby modes survive as solutions of the linearized equation $(0.1)_{\rho=0}$. They appear only if the Coriolis acceleration is not constant, i.e. if $\beta \neq 0$. The Rossby waves are responsible for most of the energy exchange in large scale motions of the atmosphere and the oceans, so they are very important for the meteorology and oceanology.

Rigorous study of small Rossby number solutions of the 3d NSE for rotating fluid in the deterministic setting was pioneered in [BMN97, BMN99] (also see the monograph [CDGG06]). There the case of constant Coriolis force is treated and no Rossby waves appear. For the case of varying Coriolis force in the deterministic setting see the work [GSR06], where a rotating shallow water model is considered and the asymptotic for small Rossby number is studied in the unbounded domain $S^{1} \times \mathbb{R}$ (accordingly the dispersion relation for Rossby waves and the resonance conditions are different from ours). In [AJW11] a version of the quasi-geostrophic 
equation on the $\beta$-plane under periodic boundary conditions was studied, with the addition of damping and deterministic forcing, and it was shown that, in the limit of small Rossby number, the flow becomes essentially zonal, i.e., independent on the vertical coordinate $y$.

The stochastic technique we employ in our study are different from the deterministic approaches in the above-mentioned works, so the results obtained differs significantly as well.

\subsection{Effective equation and main results}

Eq. (0.1) is a hamiltonian PDE. ${ }^{4}$ Accordingly, eq. (0.2) is a damped-driven hamiltonian PDE, written in the slow time. To see this, note that being re-written using the fast time $T=\beta t$ the equation becomes

$$
(-\Delta+K) \psi_{T}(T, \mathbf{x})-\rho \nu J(\psi, \Delta \psi)-\psi_{x}=\sqrt{\nu} \frac{d}{d T} \sum_{\mathbf{k} \in \mathbb{Z}_{*}^{2}} d_{\mathbf{k}} \tilde{\boldsymbol{\beta}}^{\mathbf{k}}(T) e^{i \mathbf{k}_{L} \cdot \mathbf{x}}-\nu\left(\kappa \Delta^{2} \psi-\Delta \psi\right),
$$

where $\nu=\beta^{-1}$ and $\left\{\tilde{\boldsymbol{\beta}}^{\mathbf{k}}(T)\right\}$ is another set of standard independent complex Wiener processes with the reality constraint, cf. [Kuk10, Kuk13, KM13]. In the just mentioned publications it was suggested to control the main statistical properties of solutions for similar equations as $\nu \rightarrow 0$ by studying suitable effective equations. Now we will derive an effective equation for (0.2), using the interaction representation, following our previous work [KM13].

The interaction representation is standard in physics to study small-amplitude solutions for nonlinear equations (including the quasi-geostrophic equation with small Rossby numbers, see [Naz11, CZ00] and [Maj03]). It consists in passing from the complex variables $v_{\mathbf{k}}(t)$ to the fast rotating variables

$$
a_{\mathbf{k}}(t)=e^{i \beta \lambda_{\mathbf{k}}^{L, K} t} v_{\mathbf{k}}(t), \quad \mathbf{k} \in \mathbb{Z}_{*}^{2},
$$

where

$$
\lambda_{\mathbf{k}}^{L, K}=-\frac{k_{x} / L}{K+\left|\mathbf{k}_{L}\right|^{2}}=-\frac{k_{x} L}{L^{2} K+k_{x}^{2}+L^{2} k_{y}^{2}}
$$

is the frequency of the linear rotation (corresponding to a Rossby wave in the linearised system $\left.(0.1)\right|_{\rho=0}$ with $\left.\beta=1\right)$. In view of (0.6), the $a$-variables satisfy the system of equations

$$
\begin{aligned}
\dot{a}_{\mathbf{k}}= & \frac{\rho}{L\left(K+\left|\mathbf{k}_{L}\right|^{2}\right)} \sum_{\mathbf{j}, \mathbf{n} \in \mathbb{Z}_{*}^{2}}\left|\mathbf{n}_{L}\right|^{2}(\mathbf{j} \times \mathbf{n}) a_{\mathbf{j}} a_{\mathbf{n}} \delta_{\mathbf{k}}^{\mathbf{j n}} \exp \left(-i \beta t\left(\lambda_{\mathbf{j}}^{L, K}+\lambda_{\mathbf{n}}^{L, K}-\lambda_{\mathbf{k}}^{L, K}\right)\right) \\
& -\gamma_{\mathbf{k}} a_{\mathbf{k}}+b_{\mathbf{k}} e^{i \beta \lambda_{\mathbf{k}}^{L, K}}{ }^{t} \dot{\boldsymbol{\beta}}^{\mathbf{k}}(t), \quad \mathbf{k} \in \mathbb{Z}_{*}^{2} .
\end{aligned}
$$

The terms, constituting the nonlinearity, oscillate fast as $\beta$ goes to infinity, unless the sum of the eigenvalues in the exponent vanishes. Jointly with the observation that the collection of processes $\left\{e^{i \beta \lambda_{\mathbf{k}}^{L, K} t} \dot{\boldsymbol{\beta}}^{\mathbf{k}}(t)\right\}$ is another set of standard independent white noises with the reality constraint, this leads to a guess that only the terms for which this sum equals zero (i.e., the resonant terms) contribute to the limiting dynamics, and that the effective equation is the following damped/driven hamiltonian system

$$
\dot{v}_{\mathbf{k}}=\frac{\rho}{L\left(K+\left|\mathbf{k}_{L}\right|^{2}\right)} \sum_{\mathbf{j}, \mathbf{n} \in \mathbb{Z}_{*}^{2}}\left|\mathbf{n}_{L}\right|^{2}(\mathbf{j} \times \mathbf{n}) v_{\mathbf{j}} v_{\mathbf{n}} \delta_{\mathbf{k}}^{\mathbf{j n}} \delta\left(\lambda_{\mathbf{k}}^{L, K \mathbf{j n}}\right)-\gamma_{\mathbf{k}} v_{\mathbf{k}}+b_{\mathbf{k}} \dot{\boldsymbol{\beta}}^{\mathbf{k}}(t),
$$

\footnotetext{
${ }^{4}$ Rather a Poisson one, but we neglect this subtlety.
} 
where $\mathbf{k} \in \mathbb{Z}_{*}^{2}$. Here we use another physical abbreviation:

$$
\delta\left(\lambda_{\mathbf{k}}^{L, K \mathbf{j n}}\right)=\left\{\begin{array}{cc}
1 & \text { if } \lambda_{\mathbf{j}}^{L, K}+\lambda_{\mathbf{n}}^{L, K}-\lambda_{\mathbf{k}}^{L, K}=0, \\
0 & \text { otherwise. }
\end{array} .\right.
$$

Effective equation (0.10) takes a particularly simple form for values of the external parameters $L$ and $K$ off a certain exceptional negligible set $\mathcal{Z}$ (for any fixed $K$ this set contains at most a countable set of $L$ 's). Outside $\mathcal{Z}$ the equation is an infinite system of stochastic equations which splits to invariant subsystems of complex dimension $\leq 3$; each of them is an integrable hamiltonian system, coupled with a Langevin thermostat. Accordingly, the hamiltonian part of the effective equation is a direct sum of integrable low-dimensional hamiltonian systems, which can be regarded as nonlinear modes. All nontrivial trajectories of each finite-dimensional subsystem are periodic. The whole hamiltonian part of the effective equation has the physically remarkable property of conserving all Sobolev norms. The effective equation is well posed and possesses a unique stationary measure $\mu_{0}$. In view of the integrability of the above-mentioned lower-dimensional hamiltonian systems, this measure may be written down explicitly.

A similar splitting of the limiting equation to uncoupled finite dimensional subsystems occurs in the averaging for the deterministic 3d NSE with constant Coriolis force (see [BMN97, BMN99, CDGG06]). In that case, which is closely related to the deterministic version of our study, the averaging is performed on the Poincaré modes of oscillation, and Rossby modes are not present. For the deterministic quasi-geostrophic equation, the splitting of the formal (as Ro $\rightarrow 0$ ) limiting equation to uncoupled small subsystems was observed in [RPK93], where the authors considered the equation on the two-sphere, and studied the structure and properties of the resonant relations, which define the terms of the effective equation (0.10) (see also the book [Kar94]).

The main result of the present paper consists in proving, following [Kuk10, Kuk13, KM13], that for $(L, K) \notin \mathcal{Z}$ the effective equation (0.10) describes main statistical properties of the actions $I\left(\psi^{\beta}(t)\right)$ of solutions $\psi^{\beta}$ with large $\beta$, where

$$
I(\psi(t))=\left\{I\left(v_{\mathbf{k}}(\psi(t))\right), \mathbf{k} \in \mathbb{Z}_{*}^{2}\right\}, \quad I\left(v_{\mathbf{k}}\right)=\frac{1}{2}\left|v_{\mathbf{k}}\right|^{2} .
$$

Namely, in Theorem 2.6 we show that the distribution of actions for solutions of the Cauchy problem for the effective equation well approximate those for solutions for the Cauchy problem for eq. $(0.2)=(0.6)$ with large $\beta$, on time-intervals of order one. While in Theorem 2.8 and Lemma 2.3 we prove that the unique stationary measure $\mu_{0}$ for eq. (0.10) describes the statistics of actions for stationary solutions for eq. (0.2) as $\beta \rightarrow \infty$, as well as the limiting statistic of actions for any solution as $t \rightarrow \infty$ and $\beta \rightarrow \infty$ :

Theorem 1. Let $(L, K) \notin \mathcal{Z}$. Then

i) Equation (0.2) has a unique stationary measure $\mu^{\beta}$, and

$$
I \circ \mu^{\beta} \rightarrow I \circ \mu^{0} \quad \text { as } \beta \rightarrow \infty,
$$

uniformly in $\kappa \in(0,1]$.

ii) Accordingly, for any solution $v^{\beta}(t)$ of $(0.2)$ with $\beta$-independent initial data $v^{\beta}(0)$ we have ${ }^{5}$

$$
\lim _{\beta \rightarrow \infty} \lim _{t \rightarrow \infty} \mathcal{D}\left(I\left(v^{\beta}(t)\right)=I \circ \mu^{0} .\right.
$$

\footnotetext{
${ }^{5}$ Our results do not imply that the convergence $\lim _{t \rightarrow \infty}\left(\mathcal{D}\left(I\left(v^{\beta}(t)\right)\right)\right.$ is uniform in $\kappa$.
} 
iii) If $B_{p}<\infty$ for some $p$, then

$$
\int e^{\varepsilon_{p}|v|_{h^{p}}^{2}} \mu^{0}(d v) \leq C_{p}<\infty, \quad|v|_{h^{p}}^{2}=\sum\left|v_{\mathbf{k}}\right|^{2}\left|\mathbf{k}_{L}\right|^{2 p},
$$

for suitable $\kappa$-independent constants $\varepsilon_{p}$ and $C_{p}$.

The unique limiting stationary measure $\mu^{0}$ describes the limiting, as $t \rightarrow \infty$ and $\beta \rightarrow \infty$, statistical equilibrium of actions for the system (0.2). In the same time, the limiting dynamics of the system is non-trivial and is described by the effective equation (0.10), whose specific form entails some interesting limiting properties for solutions of eq. (0.2). In particular, the nonlinear periodic modes of the hamiltonian part of the effective equation should be observable as approximate solutions of eq. (0.2) with large $\beta$ and large energy (i.e., with large $\rho$ ), and should be relevant for meteorology and physics of plasma, where equation (0.2) appears. ${ }^{6}$

Note that assertion iii) of the theorem implies that under the limit $\beta \rightarrow \infty$ no cascade of energy occurs in eq. (0.2), and that this happens uniformly in $\kappa>0$. The effective equation still depends on the parameters $L, K$ and $\kappa$, but this dependence is regular. In Proposition 2.4 we show that the iterated $\operatorname{limits}_{\lim } \lim _{\rho \rightarrow \infty} \lim _{\beta \rightarrow \infty}$ and $\lim _{\kappa \rightarrow 0} \lim _{\beta \rightarrow \infty}$ for solutions of $(0.2)$ are straightforward and the limiting systems are similar to the effective equation. In particular, they also exhibit no cascade of energy.

In Appendix we discuss what happens if the boundary condition $\mathbf{x} \in \mathbb{T}_{L, 1}^{2}$ is replaced with

$$
\mathbf{x} \in \mathbb{T}_{L \theta, \theta}^{2}=\mathbb{R} /(2 \pi L \theta \mathbb{Z}) \times \mathbb{R} /(2 \pi \theta \mathbb{Z})=: T_{\theta}^{2},
$$

where $\theta>0$ measures the size of the box of periods. In this case, again, for any $\theta$ and for a typical $L$ the limit $\beta \rightarrow \infty$ is described by the same effective equation, which is the hamiltonian system (2.16), perturbed by a Langevin thermostat in each mode $\mathbf{k}$, and the iterated limit of large box $\lim _{\theta \rightarrow \infty} \lim _{\beta \rightarrow \infty}$ still exhibits no cascade of energy.

Our treatment of equation (0.2) follows the approach to the damped-driven NLS equation on the torus $\mathbb{T}_{\theta}^{d}=\mathbb{R}^{d} / 2 \pi \theta \mathbb{Z}^{d}$, developed in our previous work [KM13, KM14]. There in paper [KM13] we derive an effective equation which describes the dynamics under a limit, similar to the limit $\beta \rightarrow \infty$ of this work. Significant analytical difference between [KM13] and what we have now comes from the fact that in [KM13] all frequencies of the unperturbed linear motion are of the form $\theta^{2}$ times an integer, so their non-zero linear combinations are $\geq \theta^{2}$ in modulus. On the contrary, now non-zero linear combinations of the frequencies may be arbitrarily small. It makes the averaging argument in the current paper (see in Section 3) more involved. The effective equation in [KM13] is significantly more complicated than the effective equation (0.8). In [KM14], using the heuristic tools, borrowed from the theory of wave turbulence, we show that under the iterated limit $\theta \rightarrow \infty$ it exhibits the power law spectra, predicted by V. Zakharov and others, in sharp difference with the results of this work.

The lack of energy cascade in eq. (0.2) under the limit when $\beta \rightarrow \infty$ and $L \rightarrow \infty$ or $\theta \rightarrow \infty$ contradicts the results of physical works, exploiting the weak turbulence approach to eq. (0.1). There power law stationary spectra and cascades of energy and enstrophy are predicted, at least in some regions of the wavenumber space. See, for instance, [Pit98] and [BN90]. There

\footnotetext{
${ }^{6}$ Note that for large $\beta$ the limiting in time dynamics of equations (0.2) with deterministic forces may be rather poor. E.g., in [AJW11] it is shown that for the deterministic $\beta$-plane equation with large $\beta$ (and the kinematic viscosity $\kappa \sim 1$ ) the global attractor for the system reduces to a single point (a suitable zonal flow).
} 
are two explanations to this contradiction. Firstly, our result applies only for typical values of the ratio $L$ between the $x$ - and $y$-periods. If $L$ takes an exceptional value, e.g. $L=1$, then the effective equation becomes more complicated; it may be that it has a power-law spectrum in analogy with the result [KM14], where we consider NLS equations on the torus $\mathbb{T}_{\theta}^{d}$. Secondly, it is not quite clear which order of limits $\beta \rightarrow \infty$ and $\theta \rightarrow \infty$ is assumed in [Pit98] and [BN90], and an order, different from what we accepted in this work, might lead to non-trivial limiting spectra.

Notation and Agreement. The stochastic terminology we use agrees with [KS91]. All filtered probability spaces we work with satisfy the usual condition (see [KS91]). ${ }^{7}$ Sometimes we forget to mention that a certain relation holds a.s.

Vectors. $\mathbb{Z}_{*}^{2}$ stands for the space of nonzero integer $2 \mathrm{~d}$ vectors. For $\mathbf{k}=\left(k_{x}, k_{y}\right) \in \mathbb{Z}_{*}^{2}, \mathbf{k}_{L}$ denotes the vector $\mathbf{k}_{L}=\left(k_{x} / L, k_{y}\right)$. For an infinite vector $\xi=\left(\xi_{\mathbf{k}}, \mathbf{k} \in \mathbb{Z}_{*}^{2}\right)$ (integer, real or complex) and $N \in \mathbb{N}$ we denote by $\xi^{N}$, depending on the context, either the finite-dimensional vector $\left(\xi_{\mathbf{k}},\left|\mathbf{k}_{L}\right| \leq N\right)$, or the infinite-dimensional vector, obtained by replacing the components $\xi_{\mathbf{k}}$ with $\left|\mathbf{k}_{L}\right|>N$ by zero.

Scalar products. The notation "." stands for the Euclidean scalar product in $\mathbb{R}^{d}$ and in $\mathbb{C}$. The latter means that if $u, v \in \mathbb{C}$, then $u \cdot v=\operatorname{Re}(\bar{u} v)$. The $L_{2}$-product is denoted $\langle\cdot, \cdot\rangle$, and we also denote by $\langle f, \mu\rangle=\langle\mu, f\rangle$ the integral of a function $f$ against a measure $\mu$.

Max/Min. For real numbers $a$ and $b$ we denote $a \vee b=\max (a, b), a \wedge b=\min (a, b)$.

Acknowledgments. We thank for discussion V. Zeitlin. This work was supported by l'Agence Nationale de la Recherche through the grant STOSYMAP (ANR 2011BS0101501).

\section{Preliminaries}

\subsection{Apriori estimates.}

In this section we discuss preliminary routine properties of solutions for eq. (0.2); most of them are well known (e.g., see [BDW98] and cf. [KS12]). By $C^{*}, C_{1}^{*}$, etc we denote various constants of the form

$$
C^{*}=C\left(L, K, B_{2},\left\|\psi_{0}\right\|_{2}\right)
$$

which occur in estimates.

It is convenient to rewrite eq. (0.2) as

$$
\begin{aligned}
\psi_{t}= & \beta(K-\Delta)^{-1} \psi_{x}+\rho(K-\Delta)^{-1} J(\psi, \Delta \psi) \\
& +\frac{d}{d t} \sum_{\mathbf{k} \in \mathbb{Z}_{*}^{2}} b_{\mathbf{k}} \boldsymbol{\beta}^{\mathbf{k}}(t) e^{i \mathbf{k}_{L} \cdot \mathbf{x}}-(K-\Delta)^{-1}\left(\kappa \Delta^{2} \psi-\Delta \psi\right),
\end{aligned}
$$

where $\psi=\psi(t, \mathbf{x})$ and $\langle\psi(t)\rangle \equiv 0$. The numbers $b_{\mathbf{k}}=d_{\mathbf{k}} /\left(K+\left|\mathbf{k}_{L}\right|^{2}\right)$ are real and even in $\mathbf{k}$. The processes $\boldsymbol{\beta}^{\mathbf{k}}$ satisfy the reality constraint, and we assume that $B_{2}<\infty$, see (0.4).

\footnotetext{
${ }^{7}$ I.e., the corresponding filtrations $\left\{\mathcal{F}_{t}\right\}$ are continuous from the right, and each $\mathcal{F}_{t}$ contains all negligible sets.
} 
By $\mathcal{H}^{p}, p \in \mathbb{R}$, we denote the Sobolev space of functions with zero mean, $\mathcal{H}^{p}=\{\psi \in$ $\left.H^{p}\left(\mathbb{T}_{L, 1}^{2}, \mathbb{R}\right),\langle\psi\rangle=0\right\}$, and denote by $\langle\cdot, \cdot\rangle$ the normalised $L^{2}$-scalar product on $\mathbb{T}_{L, 1}^{2}$,

$$
\langle u, v\rangle=\left(4 \pi^{2} L\right)^{-1} \int_{\mathbb{T}_{L, 1}^{2}} u(x) \bar{v}(x) d x .
$$

We provide $\mathcal{H}^{p}$ with the homogeneous norm $\|\cdot\|_{p}$,

$$
\|\psi\|_{p}^{2}=\left\langle(-\Delta)^{p} \psi, \psi\right\rangle=\sum_{\mathbf{k} \in \mathbb{Z}_{*}^{2}}\left|v_{\mathbf{k}}\right|^{2}\left|\mathbf{k}_{L}\right|^{2 p} \quad \text { for } \psi(\mathbf{x})=\sum_{\mathbf{k} \in \mathbb{Z}_{*}^{2}} v_{\mathbf{k}} e^{i \mathbf{k}_{L} \cdot \mathbf{x}} .
$$

In the space of complex sequences we introduce the norms

$$
|v|_{h^{m}}^{2}=\sum_{\mathbf{k} \in \mathbb{Z}_{*}^{2}}\left|v_{\mathbf{k}}\right|^{2}\left|\mathbf{k}_{L}\right|^{2 m}, \quad m \in \mathbb{R}
$$

and set $h^{m}=\left\{\left.v|| v\right|_{h^{m}}<\infty\right\}$. Then the Fourier transform

$$
\psi(\mathbf{x}) \mapsto v=\left\{v_{\mathbf{k}}\right\}_{\mathbf{k} \in \mathbb{Z}_{*}^{2}} \in \mathbb{C}^{\infty}
$$

defines isometries of the spaces $\mathcal{H}^{m}$ and $h^{m}, m \in \mathbb{R}$.

Let $\psi(t, x)$ be a solution of (1.1) such that $\psi(0, x)=\psi_{0}$. It satisfies standard a-priori estimates which we now discuss. Firstly, we fix any positive $\varepsilon_{0}$ such that $\varepsilon_{0}\left(K^{2} B_{0}+2 K B_{1}+\right.$ $\left.B_{2}\right) \leq 1 / 2$, apply the Ito formula to $f(\psi)=e^{\varepsilon_{0}\left(\|\psi\|_{2}^{2}+K\|\psi\|_{1}^{2}\right)}$ and get that

$$
\begin{aligned}
d f= & \varepsilon_{0} f\left(2\left\langle\Delta \psi,\left(-\beta \psi_{x}-\rho J(\psi, \Delta \psi)+\kappa \Delta^{2} \psi-\Delta \psi\right)\right\rangle\right) d t+d M(t) \\
& +f\left(2 \varepsilon_{0} \sum_{\mathbf{k}}\left|\mathbf{k}_{L}\right|^{2}\left(K+\left|\mathbf{k}_{L}\right|^{2}\right) b_{\mathbf{k}}^{2}+4 \varepsilon_{0}^{2} \sum_{\mathbf{k}} b_{\mathbf{k}}^{2}\left|\mathbf{k}_{L}\right|^{4}\left(K+\left|\mathbf{k}_{L}\right|^{2}\right)^{2}\left|v_{\mathbf{k}}\right|^{2}\right) d t \\
= & \varepsilon_{0} f\left(-2 \kappa\|\psi\|_{3}^{2}-2\|\psi\|_{2}^{2}\right) d t+d M(t) \\
& +\varepsilon_{0} f\left(\left(K B_{1}+B_{2}\right)+2 \varepsilon_{0} \sum_{\mathbf{k}} b_{\mathbf{k}}^{2}\left|\mathbf{k}_{L}\right|^{4}\left(K+\left|\mathbf{k}_{L}\right|^{2}\right)^{2}\left|v_{\mathbf{k}}\right|^{2}\right) d t,
\end{aligned}
$$

where $M(t)$ is a stochastic integral. Since $\varepsilon_{0} b_{\mathbf{k}}^{2}\left(K+\left|\mathbf{k}_{L}\right|^{2}\right)^{2} \leq \varepsilon_{0}\left(K^{2} B_{0}+2 K B_{1}+B_{2}\right) / 2 \leq 1 / 4$ for each $\mathbf{k}$, then taking the expectation and integrating, we obtain

$$
\begin{aligned}
\mathbf{E} f(\psi(T))-f\left(\psi_{0}\right) & \leq \varepsilon_{0} \mathbf{E} \int_{0}^{T} f(\psi(t))\left(-\|\psi(t)\|_{2}^{2}+2\left(K B_{1}+B_{2}\right)\right) d t \\
& \leq \frac{\varepsilon_{0}}{2} \int_{0}^{T}\left(-\mathbf{E} f(\psi(t))+C^{*^{\prime}}\right) d t .
\end{aligned}
$$

So the Gronwall lemma implies that, uniformly in $\beta>0, \rho>0$ and $\kappa \in(0,1]$ we have

$$
\mathbf{E} e^{\varepsilon_{0}\|\psi(t)\|_{2}^{2}} \leq C^{*} \quad \forall t \geq 0
$$


Now let us apply the Ito formula to $g(\psi)=\|\psi\|_{2}^{2}+K\|\psi\|_{1}^{2}$. We get that

$$
\begin{aligned}
& g(\psi(t))-g\left(\psi_{0}\right)+2 \int_{0}^{t}\left(\kappa\|\psi\|_{3}^{2}+\|\psi\|_{2}^{2}\right) d s=2 t \sum_{\mathbf{k}}\left|\mathbf{k}_{L}\right|^{2}\left(\left|\mathbf{k}_{L}\right|^{2}+K\right) b_{\mathbf{k}}^{2}+M^{\prime}(t), \\
& M^{\prime}(t)=2 \int_{0}^{t}\left\langle-\Delta \psi, \sum_{\mathbf{k}} d_{\mathbf{k}} e^{i \mathbf{k}_{L} \cdot \mathbf{x}} d \boldsymbol{\beta}^{\mathbf{k}}(s)\right\rangle=2 \int_{0}^{t} \sum_{\mathbf{k}} \psi_{\mathbf{k}}\left|\mathbf{k}_{L}\right|^{2} d_{\mathbf{k}} d \beta^{\mathbf{k}}(s) .
\end{aligned}
$$

By the Burkholder-Davis-Gundy inequality (see [DZ96]) for $p \geq 1$ we have

$$
Z_{T}^{p}:=\mathbf{E} \sup _{0 \leq t \leq T}\left|M^{\prime}(t)\right|^{p} \leq C_{p} \mathbf{E}\left(\int_{0}^{T}\left(\sum\left|\psi_{\mathbf{k}}\right|^{2}\left|\mathbf{k}_{L}\right|^{4} d_{\mathbf{k}}^{2}\right) d s\right)^{p / 2}
$$

On account of the Hölder inequality the r.h.s. is smaller than

$$
C_{p} T^{(p / 2-1) \vee 0} \int_{0}^{T} \mathbf{E}\left(\sum\left|\psi_{\mathbf{k}}\right|^{2}\left|\mathbf{k}_{L}\right|^{4} d_{\mathbf{k}}^{2}\right)^{p / 2} d s .
$$

Since $d_{\mathbf{k}}^{2}=b_{\mathbf{k}}^{2}\left(K+\left|\mathbf{k}_{L}\right|^{2}\right) \leq \frac{1}{2}\left(B_{1}+K B_{0}\right)$, then in view of $(1.3)$

$$
Z_{T}^{p} \leq T^{(p / 2) \vee 1} C_{1}^{*} \quad \forall p \geq 1
$$

Therefore

$$
\mathbf{E} \sup _{0 \leq t \leq T}\|\psi(t)\|_{2}^{2 p} \leq\left(T^{p}+1\right) C_{p}^{*} \quad \forall p \geq 1 .
$$

Equation (0.2), written as (0.6), is similar to the stochastic 2d Navier-Stokes equation, written for the stream function. In view of the obtained a priori estimates, the techniques usually employed to study the latter (see in [KS12]) imply the well-posedness of the initial value problem for eq. (1.1), as well as the existence and uniqueness of the stationary measure. We recall that we always assume $(0.3)$ and that $B_{2}<\infty$.

Theorem 1.1. For any $\psi_{0} \in \mathcal{H}^{2}$, eq. (0.2), supplemented with the initial condition $\psi(0)=\psi_{0}$, has a unique strong solution in $\mathcal{H}^{2}$, satisfying (1.5). Moreover, this equation has a unique stationary measure, and the corresponding stationary solution $\psi$ also satisfies estimates (1.3)(1.5), where the constants $C^{*}, C_{1}^{*}$ depend on $L, K$ and $B_{2}$.

We will need an estimate for the mapping $J: \psi \mapsto J(\psi, \Delta \psi)$.

Lemma 1.2. For any $q>3$, J defines a bounded quadratic mapping from $\mathcal{H}^{1}$ to $\mathcal{H}^{-q}$.

Proof. By the definition of stream function $\psi$, the velocity $u(\mathbf{x})$ equals $u=\nabla^{\perp} \psi$, where $\nabla^{\perp} g(\mathbf{x})=\left(-g_{y}, g_{x}\right)$. Besides, the vorticity $\omega=\nabla \times u$ equals $\Delta \psi$, so that

$$
J(\psi)=(u \cdot \nabla) \omega
$$

Let $B(u)$ be the nonlinearity of the 2 -d Navier-Stokes equation, i.e., $B(u)=(u \cdot \nabla) u$. Then, as is well known, $(u \cdot \nabla) \omega=\nabla \times B(u)$. On the other hand, $B$ defines a bounded quadratic 
map from $L^{2}$ to $\mathcal{H}^{-a}$, for any $a>2$. Indeed, since $\langle B(u), v\rangle=-\langle(u \cdot \nabla) v, u\rangle$ if $v(\mathbf{x})$ is a smooth vector field, then

$$
|\langle B(u), v\rangle| \leq C|u|_{L^{2}}^{2}|\nabla v|_{L_{\infty}} \leq C_{1}|u|_{L^{2}}^{2}\|v\|_{a},
$$

for $a>2$; so $\|B(u)\|_{-a} \leq C_{a}|u|_{L^{2}}^{2}$. Accordingly, $J$ is the composition of the following mappings: 1) $\psi \mapsto u=\nabla^{\perp} \psi$;

2) $u \mapsto w=B(u)$;

3) $w \mapsto \nabla \times w$.

The first map sends $\mathcal{H}^{1}$ to $L^{2}$, the second sends $L^{2}$ to $\mathcal{H}^{-a}, a>2$, the third sends $\mathcal{H}^{-a}$ to $\mathcal{H}^{-a-1}$. This concludes the proof.

\subsection{Study of the three-waves resonances}

Here we study the three-waves resonances for the linearized system $\left.(0.1)\right|_{\rho=0}$ :

$$
\lambda_{\mathbf{j}}^{L, K}+\lambda_{\mathbf{n}}^{L, K}=\lambda_{\mathbf{k}}^{L, K}, \quad \text { where } \mathbf{k}=\mathbf{j}+\mathbf{n} .
$$

We say that the linearised system is strongly resonant for a given value of the period $L$ and of the Froude number $K$ (and also say that the pair $(L, K)$ is strongly resonant) if (1.6) has a solution such that all three frequencies $\lambda_{\mathbf{j}}^{L, K}, \lambda_{\mathbf{n}}^{L, K}$ and $\lambda_{\mathbf{k}}^{L, K}$ do not vanish; that is the numbers $j_{x}, n_{x}$ and $k_{x}$ all are non-zero. We denote by $\mathcal{Z}$ the set, formed by all strongly resonant pairs $(L, K)$, and have the following

Lemma 1.3. For any fixed $K \geq 0$ the set of all periods $\left\{L \in \mathbb{R}^{+}:(L, K) \in \mathcal{Z}\right\}$ is at most countable.

Proof. Relation (1.6) is equivalent to equating to zero a quadratic polynomial of $L^{2}$. The polynomial's coefficients are functions of $\mathbf{j}$ and $\mathbf{n}$, and if $j_{x} \neq 0, n_{x} \neq 0$ and $k_{x} \neq 0$, then the polynomial is non-trivial. In order to see this note that the constant coefficient of the polynomial is proportional to

$$
j_{x} n_{x} k_{x}\left(n_{x} k_{x}+j_{x} k_{x}-j_{x} n_{x}\right)=j_{x} n_{x} k_{x}\left(k_{x}^{2}+n_{x}^{2}-k_{x} n_{x}\right)>0 \quad \text { if } j_{x}, n_{x}, k_{x} \neq 0,
$$

where the equality holds in view of conditions (0.7). Thus, for any fixed pair of integer vectors $\mathbf{j}, \mathbf{n}$ there exists at most two values of $L$ for which the resonance relation is satisfied. So the set of resonant $L$ 's is at most countable.

Similar results often hold also when the parameters $K$ and $L$ are not independent. A case of particular relevance for the meteorology is when $K=c L^{2}$ :

Lemma 1.4. If $K=c L^{2}$, then the set $\left\{L \in \mathbb{R}^{+}:(L, K) \in \mathcal{Z}\right\}$ is at most countable.

When $(K, L) \notin \mathcal{Z}$, eq. (1.6) still has solutions, where some of the three frequencies vanish. They are called weak resonances. The weak resonances form three groups, depending on which of the frequencies equals to zero:
i) $j_{x}=-n_{x}, j_{y}=n_{y}$,
ii) $j_{x}=0, j_{y}=-2 n_{y}$,
iii) $n_{x}=0, n_{y}=-2 j_{y}$. 


\subsection{Resonant averaging}

For a vector $v \in \mathbb{R}^{N}$, where $N \leq \infty$, we will denote $|v|_{1}=\sum\left|v_{j}\right| \leq \infty$. Given a vector $W \in \mathbb{R}^{n}$, $1 \leq n<\infty$, and a positive integer $m$, we call the set

$$
\mathcal{A}=\mathcal{A}(W, m):=\left\{s \in \mathbb{Z}^{n}:|s|_{1} \leq m, W \cdot s=0\right\}
$$

the set of resonances for $W$ of order $m$. We denote by $\mathcal{A}^{Z}$ the $\mathbb{Z}$-module in $\mathbb{Z}^{n}$, generated by $\mathcal{A}$ (called the resonance module), denote its rank by $r$ and set $\mathcal{A}^{R}=\operatorname{span} \mathcal{A}$ (so $\operatorname{dim} \mathcal{A}^{R}=r$ ). Here and everywhere below the finite-dimensional vectors are regarded as column-vectors and "span" indicates the linear envelope over real numbers.

The following fundamental lemma provides the space $\mathcal{A}^{R}$ with a very convenient integer basis. For its proof see, for example, [Bou71], Section 7:

Lemma 1.5. There exists a system $\zeta^{1}, \ldots, \zeta^{n}$ of integer vectors in $\mathbb{Z}^{n}$ such that $\operatorname{span}\left\{\zeta^{1}, \ldots, \zeta^{r}\right\}=$ $\mathcal{A}^{R}$, and the $n \times n$ matrix $R=\left(\zeta^{1} \zeta^{2} \ldots \zeta^{n}\right)$ is unimodular (i.e., $\operatorname{det} R= \pm 1$ ).

The $\mathbb{Z}$-module, generated by $\zeta^{1}, \ldots, \zeta^{r}$, contains $\mathcal{A}^{Z}$ and may be bigger than $\mathcal{A}^{Z}$, but the factor-group of the former by the latter always is finite.

We will write vectors $y \in \mathbb{R}^{n}$ as $y=\left(\begin{array}{c}y^{I} \\ y^{I I}\end{array}\right), y^{I} \in \mathbb{R}^{r}, y^{I I} \in \mathbb{R}^{n-r}$. Then clearly $\mathcal{A}^{R}=$ $\left\{R\left(\begin{array}{c}y^{I} \\ 0\end{array}\right): y^{I} \in \mathbb{R}^{r}\right\}$. Therefore

$$
s \in \mathcal{A} \Rightarrow R^{-1} s=\left(\begin{array}{c}
y^{I} \\
0
\end{array}\right) \text { for some } y^{I} \in \mathbb{Z}^{r} .
$$

Since $s \in \mathcal{A}^{R}$ implies that $W \cdot s=0$, then also

$$
\left\{s \in \mathbb{Z}^{n},|s|_{1} \leq m \text { and } R^{-1} s=\left(\begin{array}{c}
y^{I} \\
0
\end{array}\right) \text { for some } y^{I} \in \mathbb{Z}^{r}\right\} \Rightarrow s \in \mathcal{A} .
$$

Let us provide $\mathbb{R}^{n}$ with the standard basis $\left\{e^{1}, \ldots, e^{n}\right\}$, where $e_{i}^{j}=\delta_{i j}, 1 \leq i \leq n$, and consider the vectors

$$
\eta^{j}=\left(R^{T}\right)^{-1} e^{j}, \quad j=r+1, \ldots, n .
$$

Then the vectors $\eta^{r+1}, \ldots, \eta^{n}$ form a basis of $\left(\mathcal{A}^{R}\right)^{\perp}$. Indeed, these $n-r$ vectors are linearly independent, and for each $j>r, s \in \mathcal{A}$ in view of (1.8) we have

$$
\left\langle s, \eta^{j}\right\rangle=\left\langle s,\left(R^{T}\right)^{-1} e^{j}\right\rangle=\left\langle R^{-1} s, e^{j}\right\rangle=0 .
$$

For a continuous function $f$ on $\mathbb{T}^{n}$ we define its resonant average of order $m$ with respect to the vector $W$ as the function ${ }^{8}$

$$
\langle f\rangle_{W}(\varphi):=\int_{\mathbb{T}^{n-r}} f\left(\varphi+\sum_{j=r+1}^{n} \theta_{j} \eta^{j}\right) d \theta,
$$

\footnotetext{
${ }^{8}$ To understand this formula, consider the mapping $\mathbb{T}^{d} \rightarrow \mathbb{T}^{d}, \varphi \mapsto \psi=R^{T} \varphi$. In the $\psi$-variables the function $f(\varphi)$ becomes $f^{\psi}(\psi)=f\left(\left(R^{T}\right)^{-1} \varphi\right)$, and the equation $\dot{\varphi}=W$ becomes $\dot{\psi}=R^{T} W$. So $\dot{\psi}_{1}=\cdots=\dot{\psi}_{r}=0$, and the averaging of $f^{\psi}$ should be $\int_{\mathbb{T}^{n-r}} f^{\psi}\left(\psi_{1}, \ldots, \psi_{r+1}+\theta_{r+1}, \ldots, \psi_{n}+\theta_{n}\right) d \theta$, which equals (1.12).
} 
where we have $\operatorname{set} d \theta_{j}:=\frac{1}{2 \pi} d \theta_{j}$. The importance of the resonant averaging is due to the resonant version of the Kronecker-Weyl theorem. In order to state it, for a continuous function $f$ on $\mathbb{T}^{n}$, $f(\varphi)=\sum_{s} f_{s} e^{i s \cdot \varphi}$, we define its degree as $\sup _{s \in \mathbb{Z}^{n}: f_{s} \neq 0}|s|_{1}$ (it is finite or infinite; in the former case $f$ is a trigonometric polynomial).

Lemma 1.6. Let $f: \mathbb{T}^{n} \rightarrow \mathbb{C}$ be a continuous function of degree at most $m$. Then

$$
\lim _{T \rightarrow \infty} \frac{1}{T} \int_{0}^{T} f(\varphi+t W) d t=\langle f\rangle_{W}(\varphi)
$$

uniformly in $\varphi \in \mathbb{T}^{n}$. The rate of convergence in the l.h.s. depends on $n, m,|f|_{C^{0}}$ and $W$.

Proof. Let us denote the 1.h.s. in (1.13) as $\{f\}(\varphi)$. As $f(\varphi)$ is a finite sum of harmonics $f_{s} e^{i s \cdot \varphi}$, where the number of the terms is $\leq C(n, m)$ and $\left|f_{s}\right| \leq|f|_{C^{0}}$ for each $s$, it suffices to prove the assertions for $f(\varphi)=e^{i s \cdot \varphi},|s| \leq m$.

i) Let $s \in \mathcal{A}$. Then $s \cdot W=0$ and $\left\{e^{i s \cdot \varphi}\right\}=e^{i s \cdot \varphi}$ since $e^{i s \cdot(\varphi+t W)} \equiv e^{i s \cdot \varphi}$. By (1.11), the integrand in (1.12) equals $e^{i s \cdot \varphi} \prod_{j=r+1}^{n} e^{i \theta_{j} \eta^{j} \cdot s}=e^{i s \cdot \varphi}$. So in this case $\left\{e^{i s \cdot \varphi}\right\}=e^{i s \cdot \varphi}=$ $\left\langle e^{i s \cdot \varphi}\right\rangle_{W}$.

ii) Now let $s \notin \mathcal{A}$. Since $|s|_{1} \leq m$, then $s \cdot W \neq 0$. Consider $\left\{e^{i s \cdot \varphi}\right\}=\lim \frac{1}{T} \int_{0}^{T} e^{i s \cdot(\varphi+t W)} d t$. The modulus of the expression under the lim-sign is $\leq 2(T|s \cdot W|)^{-1}$. Therefore $\left\{e^{i s \cdot \varphi}\right\}=0$ and the rate of convergence to zero depends only on $\min \left\{|s \cdot W|: s \cdot W \neq 0,|s|_{1} \leq m\right\}$. By (1.9), $\left(R^{T}\right)^{-1} s \cdot e^{j} \neq 0$ for some $j>r$. So the integrand in (1.12) is a function $C e^{i \xi \cdot \theta}$, where $\xi$ is a non-zero integer vector, and $\left\langle e^{i s \cdot \varphi}\right\rangle_{W}=0$. We see that in this case (1.13) also holds.

The proof above also demonstrates that if $f$ is a finite trigonometrical polynomial $f(\varphi)=$ $\sum f_{s} e^{i s \cdot \varphi}$ of a degree at most $m$, then

$$
\langle f\rangle_{W}(\varphi)=\sum f_{s} \delta_{0, s \cdot W} e^{i s \cdot \varphi}=\sum_{s \in \mathcal{A}(W, m)} f_{s} e^{i s \cdot \varphi} .
$$

\section{$2 \quad$ Averaging for equation (1.1)}

From now on, the pair of parameters $(K, L)$ is fixed to some strongly nonresonant value, i.e. $(K, L) \notin \mathcal{Z}$, and we will usually discard $K$ and $L$ from the notation, except for Section 2.2.

\subsection{Equation (1.1) in the $v$-variables, interaction representation and effective equation}

Let us pass in eq. (1.1) with $\psi \in \mathcal{H}^{r}, r>0$, to the $v$-variables:

$$
d v_{\mathbf{k}}-i \beta \lambda_{\mathbf{k}} v_{\mathbf{k}} d t=P_{\mathbf{k}}(v) d t-\gamma_{\mathbf{k}} v_{\mathbf{k}} d t+b_{\mathbf{k}} d \boldsymbol{\beta}^{\mathbf{k}}(t), \quad \mathbf{k} \in \mathbb{Z}_{*}^{2}
$$

where $v(0)=\mathcal{F}\left(\psi_{0}\right)=: v_{0}(\mathrm{cf}$. (0.6)). Here $P$ denotes the quadratic nonlinearity of eq. (1.1) $\psi \mapsto(K-\Delta)^{-1} J(\psi, \Delta \psi)$, written in the $v$-variables. By Lemma 1.2 ,

$$
|P(v)|_{h^{s}} \leq C_{s}|v|_{h^{1}}^{2} \quad \text { if } s<-1
$$


Note (see (0.6)) that

$$
P_{\mathbf{k}}(v)=\frac{\rho}{L\left(K+\left|\mathbf{k}_{L}\right|^{2}\right)} \sum_{\mathbf{j}, \mathbf{n} \in \mathbb{Z}_{*}^{2}}\left|\mathbf{n}_{L}\right|^{2}(\mathbf{j} \times \mathbf{n}) v_{\mathbf{j}} v_{\mathbf{n}} \delta_{\mathbf{k}}^{\mathbf{j n}} .
$$

We define the resonant part $R_{\mathbf{k}}(v)$ of $P_{\mathbf{k}}(v)$ and its nonresonant part $R_{\mathbf{k}}^{n r}(v)$ as

$$
R_{\mathbf{k}}(v):=\frac{\rho}{L\left(K+\left|\mathbf{k}_{L}\right|^{2}\right)} \sum_{\mathbf{j}, \mathbf{n} \in \mathbb{Z}_{*}^{2}}\left|\mathbf{n}_{L}\right|^{2}(\mathbf{j} \times \mathbf{n}) v_{\mathbf{j}} v_{\mathbf{n}} \delta_{\mathbf{k}}^{\mathbf{j n}} \delta\left(\lambda_{\mathbf{k}}^{\mathbf{j n}}\right),
$$

and

$$
R_{\mathbf{k}}^{n r}(v):=P_{\mathbf{k}}(v)-R_{\mathbf{k}}(v)=\frac{\rho}{L\left(K+\left|\mathbf{k}_{L}\right|^{2}\right)} \sum_{\substack{\mathbf{j}, \mathbf{n} \in \mathbb{Z}_{*}^{2} \\ \lambda_{\mathbf{j}}+\lambda_{\mathbf{n}}-\lambda_{\mathbf{k}} \neq 0}}\left|\mathbf{n}_{L}\right|^{2}(\mathbf{j} \times \mathbf{n}) v_{\mathbf{j}} v_{\mathbf{n}} \delta_{\mathbf{k}}^{\mathbf{j n}} .
$$

Motivated by the averaging theory of [KM13] (see also [Kuk10, Kuk13] for the nonresonant case), we consider the following effective equation:

$$
d v_{\mathbf{k}}=R_{\mathbf{k}}(v) d t-\gamma_{\mathbf{k}} v_{\mathbf{k}} d t+b_{\mathbf{k}} d \boldsymbol{\beta}^{k}, \quad \mathbf{k} \in \mathbb{Z}_{*}^{2} .
$$

Our goal is to show that the effective equation describes the limiting, as $\beta \rightarrow 0$, dynamics of (1.1), written in the $a$-variables of the interaction representation (0.8). Indeed, let $\psi^{\beta}(t)$ be a solution of eq. (1.1), satisfying $\psi(0)=\psi_{0}$. Denote $v^{\beta}(t)=\mathcal{F}\left(\psi^{\beta}(t)\right)$ and consider the vector of $a$-variables

$$
a^{\beta}(t)=\left\{a_{\mathbf{k}}^{\beta}(t)=e^{i \beta \lambda_{\mathbf{k}} t} v_{\mathbf{k}}^{\beta}(t), \mathbf{k} \in \mathbb{Z}_{*}^{2}\right\}
$$

(cf. (0.8)). Notice that

$$
\left|v_{\mathbf{k}}^{\beta}(t)\right| \equiv\left|a_{\mathbf{k}}^{\beta}(t)\right| \quad \forall \mathbf{k}, \quad\left|v^{\beta}(t)\right|_{h^{p}} \equiv\left|a^{\beta}(t)\right|_{h^{p}} \forall p .
$$

From (2.1) we obtain the following system of equations for the vector $a^{\beta}(t)$ :

$$
d a_{\mathbf{k}}^{\beta}=\left(\mathbf{R}_{\mathbf{k}}\left(a^{\beta}, \beta t\right)-\gamma_{\mathbf{k}} a_{\mathbf{k}}^{\beta}\right) d t+b_{\mathbf{k}} e^{i \beta_{\mathbf{k}} \lambda_{\mathbf{k}} t} d \boldsymbol{\beta}^{\mathbf{k}}(t), \quad \mathbf{k} \in \mathbb{Z}_{*}^{2},
$$

where $\mathbf{R}=\left(\mathbf{R}_{\mathbf{k}}, \mathbf{k} \in \mathbb{Z}_{*}^{2}\right)$, is the nonlinearity $P$, written in the $a$-variables. By (2.2) and (2.7),

$$
|\mathbf{R}(a, \tau)|_{h^{s}} \leq C_{s}|a|_{h^{1}}^{2} \quad \text { if } s<-1,
$$

for each $\tau$. We see immediately that $\mathbf{R}_{\mathbf{k}}=R_{\mathbf{k}}\left(a^{\beta}\right)+\mathcal{R}_{\mathbf{k}}\left(a^{\beta}, \beta t\right)$, where

$$
\begin{aligned}
\mathcal{R}_{\mathbf{k}}(a, \beta t) & =\left.e^{i \beta \lambda_{\mathbf{k}} t} R_{\mathbf{k}}^{n r}(v)\right|_{v_{\mathbf{k}}:=e^{-i \beta \lambda_{\mathbf{k}} t} a_{\mathbf{k}} \forall \mathbf{k}} \\
& =\frac{\rho}{L\left(K+\left|\mathbf{k}_{L}\right|^{2}\right)} \sum_{\substack{\mathbf{j}, \mathbf{n} \in \mathbb{Z}_{*}^{2} \\
\lambda_{\mathbf{j}}+\lambda_{\mathbf{n}}-\lambda_{\mathbf{k}} \neq 0}}\left|\mathbf{n}_{L}\right|^{2}(\mathbf{j} \times \mathbf{n}) a_{\mathbf{j}} a_{\mathbf{n}} \delta_{\mathbf{k}}^{\mathbf{j n}} e^{-i \beta t\left(\lambda_{\mathbf{j}}+\lambda_{\mathbf{n}}-\lambda_{\mathbf{k}}\right)} .
\end{aligned}
$$

Since the numbers $\lambda_{\mathbf{k}}$ are odd in $\mathbf{k}$, then the collection of processes $\left\{\overline{\boldsymbol{\beta}}^{\mathbf{k}}(t):=\int e^{i \beta \lambda_{\mathbf{k}} t} d \boldsymbol{\beta}^{\mathbf{k}}(t), \mathbf{k} \in\right.$ $\left.\mathbb{Z}_{*}^{2}\right\}$ is another set of standard independent complex Wiener processes with the reality constraint. So the vector-process $a^{\beta}(t)$ is a weak solution of the system of equations

$$
d a_{\mathbf{k}}^{\beta}=\left(R_{\mathbf{k}}\left(a^{\beta}\right)+\mathcal{R}_{\mathbf{k}}\left(a^{\beta}, \beta t\right)-\gamma_{\mathbf{k}} a_{\mathbf{k}}^{\beta}\right) d t+b_{\mathbf{k}} d \boldsymbol{\beta}^{\mathbf{k}}(t), \quad \mathbf{k} \in \mathbb{Z}_{*}^{2} .
$$

This system is identical to the effective equation (2.6), apart from terms which oscillate fast as $\beta \rightarrow \infty$. 


\subsection{Properties of the effective equation. Limits $L=\rho \rightarrow \infty$ and $\varkappa \rightarrow 0$}

To write the effective equation explicitly we consider separately the variables $v_{\mathbf{k}}$ with $k_{x}=0$ and $k_{x} \neq 0$. Since the pair of parameters $(K, L)$ is not strongly resonant, then when $k_{x}=0$, the only terms which survive in $R_{\mathbf{k}}(v)$ are those where $\mathbf{j}$ and $\mathbf{n}$ satisfy the relation i) of Section 1.2, while for $k_{x} \neq 0$ only the terms falling in the cases ii)-iii) give contribution. For the case $k_{x}=0$, the nonlinearity vanishes if $k_{y}$ is odd, while if it is even, then

$$
R_{\mathbf{k}}(v)=\frac{\rho}{L\left(K+\left|\mathbf{k}_{L}\right|^{2}\right)} \sum_{j_{x} \in \mathbb{Z}}\left(\frac{j_{x}^{2}}{L^{2}}+\frac{k_{y}^{2}}{4}\right) j_{x} k_{y} v_{\left(j_{x}, k_{y} / 2\right)} v_{\left(-j_{x}, k_{y} / 2\right)}, \quad k_{x}=0,
$$

which in turn vanishes because the odd symmetry in $j_{x}$. On the other hand, if $k_{x} \neq 0$, then we have the case ii) or iii), when $\mathbf{j}$ and $\mathbf{n}$ are completely determined by $\mathbf{k}$. So the sum in (2.4) contains only two terms, and we get that

$$
R_{\mathbf{k}}(v)=\left(2 \rho L \frac{k_{x} k_{y}}{k_{x}^{2}+L^{2} k_{y}^{2}+L^{2} K}\left(3 k_{y}^{2}-\frac{k_{x}^{2}}{L^{2}}\right) v_{\overline{\mathbf{k}}} v_{\left(0,2 k_{y}\right)}\right),
$$

where we denoted $\overline{\mathbf{k}}:=\left(k_{x},-k_{y}\right)$. Note that this formula applies both for the case $k_{x}=0$ and the case $k_{x} \neq 0$. We immediately see that

$$
\left|R_{\mathbf{k}}(v)\right| \leq C^{*} k_{x} k_{y}\left|v_{\overline{\mathbf{k}}}\right|\left|v_{\left(0,2 k_{y}\right)}\right| \leq C^{*^{\prime}}(\mathbf{k}, s)|v|_{h^{s}}|v|_{h^{s}}
$$

so $R_{\mathbf{k}}$ defines a bounded quadratic function on $h^{s}$, for any $\mathbf{k}$ and any $s$. In a similar way, for any $s$ one has

$$
|R(v)|_{h^{s}} \leq C^{*}|v|_{h^{1}}|v|_{h^{s+1}} .
$$

More importantly, the expression (2.11) shows that the hamiltonian part of the effective equation,

$$
\dot{v}_{\mathbf{k}}=R_{\mathbf{k}}(v), \quad \mathbf{k} \in \mathbb{Z}_{*}^{2},
$$

is integrable and decomposes to invariant subsystems of complex dimension at most three. Indeed, if $k_{x}$ or $k_{y}$ vanishes, then $R_{\mathbf{k}}=0$ and $v_{\mathbf{k}}(t)=$ const.

Now let $k_{x}, k_{y} \neq 0$. If $3 L^{2} k_{y}^{2}=k_{x}^{2}$, then again the equation for $v_{\mathbf{k}}$ trivialises. ${ }^{9}$ Suppose that $3 L^{2} k_{y}^{2} \neq k_{x}^{2}$ and denote

$$
A_{\mathbf{k}}=2 \rho L \frac{k_{x} k_{y}}{k_{x}^{2}+L^{2} k_{y}^{2}+L^{2} K}\left(3 k_{y}^{2}-\frac{k_{x}^{2}}{L^{2}}\right) \in \mathbb{R} .
$$

Then $A_{\overline{\mathbf{k}}} \equiv-A_{\mathbf{k}}$. Eq. (2.14) (with any fixed $\mathbf{k}$ ) belongs to the following invariant sub-system of $(2.14)$ :

$$
\begin{aligned}
& \dot{v}_{\mathbf{k}}=A_{\mathbf{k}} v_{\left(0,2 k_{y}\right)} v_{\overline{\mathbf{k}}}, \\
& \dot{v}_{\overline{\mathbf{k}}}=-A_{\mathbf{k}} \bar{v}_{\left(0,2 k_{y}\right)} v_{\mathbf{k}}, \\
& \dot{v}_{\left(0,2 k_{y}\right)}=0
\end{aligned}
$$

\footnotetext{
${ }^{9}$ This case is non-typical and may be excluded by removing another countable set of parameters $L$.
} 
(we recall that $v_{\left(0,2 k_{y}\right)}=\bar{v}_{\left(0,-2 k_{y}\right)}$ by the reality condition $(0.5)$ ). This system is explicitly soluble: if $v_{\left(0,2 k_{y}\right)}(0) \neq 0$, then

$$
\begin{aligned}
& v_{\left(0,2 k_{y}\right)}(t)=\text { Const }, \\
& v_{\mathbf{k}}(t)=v_{\mathbf{k}}(0) \cos \left(\left|A_{\mathbf{k}} v_{\left(0,2 k_{y}\right)}\right| t\right)+v_{\overline{\mathbf{k}}}(0) \operatorname{sgn}\left(A_{\mathbf{k}} v_{\left(0,2 k_{y}\right)}\right) \sin \left(\left|A_{\mathbf{k}} v_{\left(0,2 k_{y}\right)}\right| t\right),
\end{aligned}
$$

where for a complex number $z$ we denote

$$
\operatorname{sgn}(z)=z /|z| \text { if } z \neq 0 \text {, and } \operatorname{sgn}(0)=0 .
$$

The formula for $v_{\overline{\mathbf{k}}}(t)$ is obtained from that for $v_{\mathbf{k}}(t)$ by swapping $\mathbf{k}$ with $\overline{\mathbf{k}}$ and replacing $v_{\left(0,2 k_{y}\right)}$ by $\bar{v}_{\left(0,2 k_{y}\right)}$. All these solutions are periodic. If $v_{\left(0,2 k_{y}\right)}=0$, then $\left(v_{\mathbf{k}}, v_{\overline{\mathbf{k}}}, v_{\left(0,2 k_{y}\right)}\right)$ is a singular point for the vector field in (2.16).

Passing in (2.16) from the variables $\left(v_{\mathbf{k}}, v_{\overline{\mathbf{k}}}\right)$ to $\left(z_{1}, z_{2}\right)$, where

$$
z_{1}=v_{\mathbf{k}}-i \operatorname{sgn}\left(A_{k} v_{\left(0,2 k_{y}\right)}\right) v_{\overline{\mathbf{k}}}, \quad z_{2}=v_{\mathbf{k}}+i \operatorname{sgn}\left(A_{k} v_{\left(0,2 k_{y}\right)}\right) v_{\overline{\mathbf{k}}},
$$

we get for $\left(z_{1}, z_{2}\right)$ equations

$$
\dot{z}_{1}=i\left|A_{\mathbf{k}} v_{\left(0,2 k_{y}\right)}\right| z_{1}, \quad \dot{z}_{2}=-i\left|A_{\mathbf{k}} v_{\left(0,2 k_{y}\right)}\right| z_{2} .
$$

Therefore the functions $\left|z_{1}\right|^{2},\left|z_{2}\right|^{2}$ and $\operatorname{sgn}^{2}\left(z_{1} z_{2}\right)$ are integrals of motion for (2.16). We have proved

Lemma 2.1. Let $k_{x}, k_{y} \neq 0$ and $3 L^{2} k_{y}^{2} \neq k_{x}^{2}$. Then the three-dimensional complex system (2.16) is an invariant subsystem for (2.14). Its singular points form the locus $\partial=\left\{v_{\left(0,2 k_{y}\right)}=\right.$ $0\} \cup\left\{v_{\mathbf{k}}=v_{\overline{\mathbf{k}}}=0\right\}$. The system has 5 real integrals of motion

$$
v_{\left(0,2 k_{y}\right)},\left|z_{1}\right|^{2},\left|z_{2}\right|^{2}, \operatorname{sgn}^{2}\left(z_{1} z_{2}\right) .
$$

Outside $\partial$ they are smooth and independent, so there the system is integrable. All its trajectories outside $\partial$ are periodic and are given by (2.17).

Similar infinite-dimensional hamiltonian systems which split into finite-dimensional subsystems systematically arise as resonant parts of various infinite-dimensional Hamiltonians. See the book [Kar10] where many examples are discussed.

It immediately follows from the lemma (and can be easily checked directly) that $\left|v_{\mathbf{k}}\right|^{2}+\left|v_{\overline{\mathbf{k}}}\right|^{2}$ and $\left|v_{\left(0,2 k_{y}\right)}\right|^{2}$ are integrals of motion for (2.16). So we have

Corollary 2.2. The quantity $|v|_{h^{m}}^{2}$ is an integral of motion for (2.14), for any $m$.

Accordingly, the effective equation (2.6) also splits into invariant subsystems of complex dimension one (if $k_{x} k_{y}=0$ or $3 L^{2} k_{y}^{2}=k_{x}^{2}$ ), or of dimension three (otherwise). These systems either are independent, or have catalytic interaction through the variables $v_{\left(0,2 k_{y}\right)}$ which satisfy the Ornstein-Uhlenbeck equation

$$
\dot{v}_{\left(0,2 k_{y}\right)}=-\gamma_{\left(0,2 k_{y}\right)} v_{\left(0,2 k_{y}\right)}+b_{\left(0,2 k_{y}\right)} \dot{\beta}^{\left(0,2 k_{y}\right)},
$$

independent from other variables. 
Using Corollary 2.2 and arguing as in Section 1.1 it is easy to get a-priori estimates for solution of the effective equation (2.6):

$$
\begin{gathered}
\mathbf{E} e^{\varepsilon_{p}|v(T)|_{h p}^{2}}-e^{\varepsilon_{p}\left|v_{0}\right|_{h^{p}}^{2}} \leq \mathbf{E} \int_{0}^{T} \varepsilon_{p} e^{\varepsilon_{p}|v(t)|_{h^{p}}^{2}}\left(2 \varepsilon_{p} B_{p}|v(t)|_{h^{p}}^{2}-2 C_{p}|v(t)|_{h^{p}}^{2}+B_{p}\right) d t \\
\leq \mathbf{E} \int_{0}^{t} \varepsilon_{p} e^{\varepsilon_{p}|v(t)|_{h^{p}}^{2}}\left(-|v(t)|_{h^{p}}^{2}+B_{p}\right) d t, \quad \text { if } B_{p}<\infty
\end{gathered}
$$

for any $\varepsilon_{p} \leq C_{p} /\left(2 B_{p}\right)$, where the constant $C_{p}$ depends on $K$ and $L$. This implies, via the Gronwall lemma, that

$$
\mathbf{E} e^{\varepsilon_{p}|v(T)|_{h^{p}}^{2}} \leq C\left(\left|v_{0}\right|_{h^{p}}, B_{p}\right) \quad \text { if } B_{p}<\infty .
$$

This analysis of equation (2.6) holds for $\kappa \in[0,1]$ (not only for $\kappa \in(0,1]$ ). Due to the decoupling of eq. (2.6) to finite-dimensional subsystems and since each subsystem is mixing, (e.g., see [Ver97]), we have

Lemma 2.3. If $\kappa \in[0,1]$ and for some $p \geq 0$ we have $B_{p}<\infty$ and $v_{0} \in h^{p}$, then a strong solution of (2.6) in the space $h^{p}$ such that $v(0)=v_{0}$ exists globally in time, is unique and satisfies (2.18). Moreover, the equation has a unique stationary measure $\mu^{0}$. It is supported by the space $h^{p}$, is mixing and $\int e^{\varepsilon_{p}|v|_{h^{p}}^{2}} \mu^{0}(d v) \leq C_{p}<\infty$ for a suitable $\kappa$-independent constants $\varepsilon_{p}$ and $C_{p}$.

As a consequence of straightforward analysis of the formula (2.11) we have:

Proposition 2.4. When $L=\rho \rightarrow \infty$ or when $\varkappa \rightarrow 0$, solutions of the effective equation (2.6) a.s. converge to solutions of the limiting system, obtained from (2.6) by replacing $L=\rho$ by $\infty$ or $\varkappa$ by 0 . Besides, the stationary measure of (2.6) weakly converges to the unique stationary measure of the corresponding limiting system.

If $\rho \gg 1$, then by Lemma 2.1 the subsystems, forming the effective equation, become fastslow systems with one fast variable. So the limit $\rho \rightarrow \infty$ for the system (2.6) can be described, using the classical stochastic averaging, see [FW84].

\subsection{Averaging theorem for the initial-value problem.}

For any $p \in \mathbb{R}$ denote $X^{p}=C\left([0, T], h^{p}\right)$.

Lemma 2.5. Let $p_{1} \leq p \leq p_{2}$ and $Q \subset X^{p}$ such that:

i) $Q$ is bounded in $X^{p_{2}}$,

ii) $Q$ is uniformly continuous in $h^{p_{1}}$

Then $Q$ is pre-compact in $X^{p}$.

Proof. We have to show that every sequence $\left\{x_{1}, x_{2}, \ldots\right\} \subset Q$ contains a subsequence $\left\{x_{n(j)}, j \geq\right.$ $1\}$ which converges in $X^{p}$ to some point $x_{*}$. For any $N \geq 1$ consider the projection

$$
\Pi_{N}: h \rightarrow h, \quad\left(v_{\mathbf{k}}, \mathbf{k} \in \mathbb{Z}_{*}^{2}\right) \mapsto v^{N},
$$


where $v_{\mathbf{k}}^{N}=v_{\mathbf{k}}$ if $|\mathbf{k}| \leq N$, and $v_{\mathbf{k}}^{N}=0$ otherwise. Then by the Ascoli-Arzelà theorem for each $N$ there is a subsequence $\left\{x_{n_{N}(j)}, j \geq 1\right\}$, such that

$$
\Pi_{N} x_{n_{N}(j)} \rightarrow y_{N} \in \Pi_{N} h \quad \text { as } \quad j \rightarrow \infty,
$$

for some $y_{N}$. Applying the diagonal process we get a subsequence $\left\{x_{n(j)}, j \geq 1\right\}$ with the property that

$$
\Pi_{N} x_{n(j)} \rightarrow y_{N} \in \Pi_{N} h \quad \text { as } \quad j \rightarrow \infty,
$$

for each $N$. Clearly

$$
\Pi_{N} y_{M}=y_{N} \quad \text { if } \quad M \geq N
$$

and by i)

$$
\left\|y_{N}\right\|_{X^{p_{2}}} \leq C \quad \forall N
$$

for a suitable $C$. By $(2.20),(2.21)$ the sequence $\left\{y_{N}, N \geq 1\right\} \subset X^{p}$ is a Cauchy sequence. So $y_{N} \rightarrow x_{*} \in X^{p}$. This convergence jointly with (2.19) and (2.20) imply that $x_{n(j)} \rightarrow x_{*}$.

Let $a^{\beta}(t)$ be a solution of (2.10) such that $a^{\beta}(0)=v_{0}=\mathcal{F}\left(\psi_{0}\right) \in h^{2}$. Denote the white noise in $(2.10)$ as $\dot{\zeta}(t, x)$ and denote $U_{1}(t)=\mathbf{R}\left(a^{\beta}, \beta t\right), U_{2}(t)=-\gamma_{k} a_{k}$. Then

$$
\dot{a}^{\beta}-\dot{\zeta}=U_{1}+U_{2} .
$$

Fix some $r<-1$. In view of (2.8), $\left\|U_{1}(s)\right\|_{r} \leq C\|\psi(s)\|_{1}^{2}$. So, by (1.5),

$$
\mathbf{E} \int_{t}^{(t+\tau) \wedge T}\left\|U_{1}\right\|_{r} d s \leq C_{1}^{*}(T+1) \tau,
$$

for any $t \in[0, T]$ and $\tau>0$. Similar,

$$
\mathbf{E} \int_{t}^{(t+\tau) \wedge T}\left\|U_{2}\right\|_{r} d s \leq C^{*} \mathbf{E} \int_{t}^{(t+\tau) \wedge T}\|\psi\|_{2} \leq C_{2}^{*}(T+1) \tau .
$$

If $U_{1}$ and $U_{2}$ are such that

$$
\int_{t}^{(t+\tau) \wedge T}\left\|U_{1}\right\|_{r} d s \leq \tau K_{1}, \quad \int_{t}^{(t+\tau) \wedge T}\left\|U_{2}\right\|_{r} d s \leq \tau K_{2}
$$

for all $t$ and $\tau$ as above, then the curve $t \mapsto \int_{0}^{t}\left(U_{1}+U_{2}\right) d s \in h^{r}$ has a modulus of continuity which depends only on $K_{1}, K_{2}$.

It is classical that

$$
\mathbf{P}\left\{\|\zeta\|_{C^{1 / 3}\left([0, T], h^{0}\right)} \leq R_{3}\right\} \rightarrow 1 \quad \text { as } \quad R_{3} \rightarrow \infty,
$$

and that the functions $\zeta$ such that $\|\zeta\|_{C^{1 / 3}\left([0, T], h^{0}\right)} \leq R_{3}$ have a modulus of continuity in $h^{0}$ which depends only on $R_{3}$.

In view of (2.22) and what was said above, for any $\varepsilon>0$ there is a set $Q_{\varepsilon}^{1} \subset X^{r}$, formed by equicontinuous functions, such that

$$
\mathbf{P}\left\{a^{\beta} \in Q_{\varepsilon}^{1}\right\} \geq 1-\varepsilon,
$$


for each $\beta$. By (1.5),

$$
\mathbf{P}\left\{\left\|a^{\beta}\right\|_{X^{2}} \geq C^{*^{\prime}} \varepsilon^{-1}\right\} \leq \varepsilon,
$$

for each $\beta$, for a suitable $C^{*^{\prime}}$. Consider the set

$$
Q_{\varepsilon}=\left\{a \in Q_{\varepsilon}^{1}:\|a\|_{X^{2}} \leq C^{*^{\prime}} \varepsilon^{-1}\right\} .
$$

Then $\mathbf{P}\left\{a^{\beta} \in Q_{\varepsilon}\right\} \geq 1-2 \varepsilon$, for each $\beta$. By this relation and Lemma 2.5 the set of laws $\left\{\mathcal{D}\left(a^{\beta}\right), 1 \leq \beta<\infty\right\}$, is tight in $X^{2-\gamma}$, for any positive $\gamma$. So by the Prokhorov theorem there is a sequence $\beta_{l} \rightarrow \infty$ and a Borel measure $\mathcal{Q}^{0}$ on $X^{2-\gamma}$ such that

$$
\mathcal{D}\left(a^{\beta_{l}}(\cdot)\right) \rightarrow \mathcal{Q}^{0} \quad \text { as } \quad \beta_{l} \rightarrow \infty,
$$

weakly in $X^{2-\gamma}$. Accordingly, due to (2.7), for the actions $I$ (see (0.12)) we have

$$
\mathcal{D}\left(I\left(v^{\beta_{l}}(\cdot)\right)\right) \rightarrow I \circ \mathcal{Q}^{0} \quad \text { as } \quad \beta_{l} \rightarrow \infty,
$$

weakly in $C\left([0, T] ; h_{I}^{2-\gamma}\right)=: X_{I}^{2-\gamma}$.

Relation (1.5) and the Fatou lemma imply that

$$
\int\|a\|_{X^{2}}^{p} \mathcal{Q}^{0}(d a) \leq C_{p}^{*} \quad \forall p>0
$$

cf. Lemma 1.2.17 in [KS12].

Theorem 2.6. Let $v^{\beta}(t)$ be a solution of (2.1) such that $v^{\beta}(0)=v_{0} \in h^{2}$, and let $\gamma$ be any positive number. Then there exists a unique weak solution a(t) of the effective equation (2.6), satisfying the $\kappa$-independent estimates $(2.25)$, such that $\mathcal{D}(a)=\mathcal{Q}^{0}, a(0)=v_{0}$ a.s., and the convergence (2.24) holds as $\beta \rightarrow \infty$. Moreover, this convergence holds uniformly in $\kappa \in(0,1]$. That is,

$$
\operatorname{dist}\left(\mathcal{D}\left(I\left(v^{\beta}(\cdot)\right), I \circ \mathcal{Q}^{0}\right) \rightarrow 0 \quad \text { as } \beta \rightarrow \infty,\right.
$$

uniformly in $\kappa \in(0,1]$, where dist is the Lipschitz-dual distance in the space of Borel measures in $X_{I}^{2-\gamma} \cdot 10$

Proof. The proof follows the Khasminski scheme (see [Kha68]), and is similar to the proof in [KM13]. The main difference compare to the argument in [KM13] is in the demonstration of the following crucial lemma:

Lemma 2.7. For any $\mathbf{k} \in \mathbb{Z}_{*}^{2}$ the following convergences hold:

$$
\begin{gathered}
\mathfrak{A}_{\mathbf{k}}^{\beta}:=\mathbf{E} \max _{0 \leq t \leq T}\left|\int_{0}^{t} \mathcal{R}_{\mathbf{k}}\left(a^{\beta}(s), \beta s\right) d s\right| \rightarrow 0 \quad \text { as } \beta \rightarrow \infty, \\
\mathbf{E} \max _{0 \leq t \leq T}\left|\int_{0}^{t} \mathcal{R}_{\mathbf{k}}\left(a^{\beta}(s), \beta s\right) d s\right|^{2} \rightarrow 0 \quad \text { as } \beta \rightarrow \infty .
\end{gathered}
$$

${ }^{10}$ This distance metrizes the weak convergence of measures in $X_{I}^{2-\gamma}$, see [KS12]. 
The proof of the lemma is given below in Section 3. Now we derive the theorem from the lemma.

For $t \in[0, T]$ and $\beta \geq 0$ consider the processes

$$
N_{\mathbf{k}}^{\beta}(t)=a_{\mathbf{k}}^{\beta}(t)-\int_{0}^{t}\left(R_{\mathbf{k}}\left(a^{\beta}(s)\right)-\gamma_{\mathbf{k}} a_{\mathbf{k}}^{\beta}(s)\right) d s, \quad \mathbf{k} \in \mathbb{Z}_{*}^{2} .
$$

We regard them as processes on the measurable space $\left(X^{2-\gamma}, \mathcal{F}, \mathbf{P}\right)$, where $\mathcal{F}$ is the Borel sigma-algebra, given the natural filtration $\left\{\mathcal{F}_{t}, 0 \leq t \leq T\right\}$, complemented by negligible sets.

Due to (2.10), where $R_{\mathbf{k}}+\mathcal{R}_{\mathbf{k}}=\mathbf{R}_{\mathbf{k}}$, we can write $N_{\mathbf{k}}^{\beta}$ as

$$
N_{\mathbf{k}}^{\beta}(t)=\widetilde{N}_{\mathbf{k}}^{\beta}(t)+\bar{N}_{\mathbf{k}}^{\beta}(t),
$$

where the process $\widetilde{N}_{\mathbf{k}}^{\beta}(t)=a^{\beta}(t)-\int_{0}^{t}\left(\mathbf{R}_{\mathbf{k}}\left(a^{\beta}(s), \beta s\right)-\gamma_{\mathbf{k}} a_{\mathbf{k}}^{\beta}(s)\right) d s$ is a martingale and the process

$$
\bar{N}_{\mathbf{k}}^{\beta}(t)=\int_{0}^{t} \mathcal{R}_{\mathbf{k}}\left(a^{\beta}(s), \beta s\right) d s
$$

should be regarded as a disparity. The convergence $\mathcal{D}\left(a^{\beta}\right) \rightarrow \mathcal{Q}^{0}$ weakly in $X^{2-\gamma}$ and Lemma 2.7 imply that the processes

$$
N_{\mathbf{k}}(t)=a_{\mathbf{k}}(t)-\int_{0}^{t}\left(R_{\mathbf{k}}(a(s))-\gamma_{\mathbf{k}} a_{\mathbf{k}}(s)\right) d s, \quad \mathbf{k} \in \mathbb{Z}_{*}^{2},
$$

are $\mathcal{Q}^{0}$ martingales (see for details [KP08], Proposition 6.3). Besides, $a(0)=v_{0}, \mathcal{Q}^{0}$-a.s.

To proceed, we re-interpret equations (2.1) and (2.10) with the reality constraint (0.5) as systems of equations for real vectors $v_{\mathbf{k}}(t) \in \mathbb{R}^{2}, a_{\mathbf{k}}(t) \in \mathbb{R}^{2}$, where

$$
\mathbf{k} \in \mathbb{Z}_{+}^{2}=\left\{\mathbf{k}=\left(k_{x}, k_{y}\right) \in \mathbb{Z}_{*}^{2}: k_{x}>0 \text {, or } k_{x}=0 \text { and } k_{y}>0\right\}
$$

(so that $\mathbb{Z}_{+}^{2} \cup-\mathbb{Z}_{+}^{2}=\mathbb{Z}_{*}^{2}$ ). The diffusion matrix for both systems is the block-matrix

$$
A=\operatorname{diag}\left\{\left(\begin{array}{cc}
b_{\mathbf{k}}^{2} & 0 \\
0 & b_{\mathbf{k}}^{2}
\end{array}\right), \mathbf{k} \in \mathbb{Z}_{+}^{2}\right\} .
$$

We will write real two-vectors as $v_{\mathbf{k}}=\left(\begin{array}{c}v_{\mathbf{k}}^{+} \\ v_{\mathbf{k}}^{-}\end{array}\right)$, and accordingly write matrix $A$ as $A=\left(A_{\mathbf{k}_{1} \mathbf{k}_{2}}^{\sigma_{1} \sigma_{2}}\right)$, $\sigma_{1}, \sigma_{2} \in\{+,-\}$, where $A_{\mathbf{k}_{1} \mathbf{k}_{2}}^{\sigma_{1} \sigma_{2}}=\delta_{\mathbf{k}_{1} \mathbf{k}_{2}} \delta_{\sigma_{1} \sigma_{2}} b_{\mathbf{k}_{1}}^{2}$.

Since the process $\left(a_{\mathbf{k}}^{\beta}(t)=\left(\begin{array}{c}a_{\mathbf{k}}^{+\beta} \\ a_{\mathbf{k}}^{-\beta}\end{array}\right) \in \mathbb{R}^{2}, \mathbf{k} \in \mathbb{Z}_{+}^{2}\right)$ satisfies (2.10), then for $\sigma_{j} \in\{+,-\}$ and $\mathbf{k}_{j} \in \mathbb{Z}_{+}^{2}$ the process

$$
\begin{aligned}
\left(a_{\mathbf{k}_{1}}^{\sigma_{1} \beta} a_{\mathbf{k}_{2}}^{\sigma_{2} \beta}\right)(t)-\left(a_{\mathbf{k}_{1}}^{\sigma_{1} \beta} a_{\mathbf{k}_{2}}^{\sigma_{2} \beta}\right)(0) & -\int_{0}^{t}\left[a_{\mathbf{k}_{1}}^{\sigma_{1} \beta}\left(R_{\mathbf{k}_{2}}^{\sigma_{2}}+\mathcal{R}_{\mathbf{k}_{2}}^{\sigma_{2}}-\gamma_{\mathbf{k}_{2}} a_{\mathbf{k}_{2}}^{\sigma_{2} \beta}\right)\right. \\
& \left.+a_{\mathbf{k}_{2}}^{\sigma_{2} \beta}\left(R_{\mathbf{k}_{1}}^{\sigma_{1}}+\mathcal{R}_{\mathbf{k}_{1}}^{\sigma_{1}}-\gamma_{\mathbf{k}_{1}} a_{\mathbf{k}_{1}}^{\sigma_{1} \beta}\right)\right] d s-A_{\mathbf{k}_{1} \mathbf{k}_{2}}^{\sigma_{1} \sigma_{2}} t
\end{aligned}
$$


where $R=R\left(a^{\beta}(t)\right)$ and $\mathcal{R}=\mathcal{R}\left(a^{\beta}(t), \beta t\right)$, is a martingale. Passing to the limit as $\beta_{l} \rightarrow \infty$, using (2.23) and (2.28), we get that the process

$$
\begin{aligned}
\left(a_{\mathbf{k}_{1}}^{\sigma_{1}} a_{\mathbf{k}_{2}}^{\sigma_{2}}\right)(t)-\left(a_{\mathbf{k}_{1}}^{\sigma_{1}} a_{\mathbf{k}_{2}}^{\sigma_{2}}\right)(0) & -\int_{0}^{t}\left[a_{\mathbf{k}_{1}}^{\sigma_{1}}\left(R_{\mathbf{k}_{2}}^{\sigma_{2}}-\gamma_{\mathbf{k}_{2}} a_{\mathbf{k}_{2}}^{\sigma_{2}}\right)\right. \\
& \left.+a_{\mathbf{k}_{2}}^{\sigma_{2}}\left(R_{\mathbf{k}_{1}}^{\sigma_{1}}-\gamma_{\mathbf{k}_{1}} a_{\mathbf{k}_{1}}^{\sigma_{1}}\right)\right] d s-A_{\mathbf{k}_{1} \mathbf{k}_{2}}^{\sigma_{1} \sigma_{2}} t
\end{aligned}
$$

is a $\mathcal{Q}^{0}$-martingale.

That is, $\mathcal{Q}^{0}$ is a solution of the martingale problem with drift $R_{\mathrm{k}}$ and diffusion $A$. Hence, $\mathcal{Q}^{0}$ is the law of a weak solution of eq. (2.6) with the initial condition $a(0)=v_{0}$. Such a solution exists for any $v_{0} \in h^{2}$, so by the uniqueness of a strong solution of the effective equation and the Yamada-Watanabe argument (see [Yor74, KS91, MR99]), weak and strong solutions for (2.6) both exist and are unique. Hence, the limit in (2.23) does not depend on the sequence $\beta_{l} \rightarrow \infty$ and the convergence holds as $\beta \rightarrow \infty$.

It remains to show that the convergence (2.26) is uniform in $\kappa$. Assume that it is not. Then there exists $\bar{\gamma}>0$ and sequences $\beta_{l} \rightarrow \infty$ and $\left\{\kappa_{l}\right\} \subset(0,1]$ such that

$$
\operatorname{dist}\left(\mathcal{D}\left(I\left(v_{\kappa_{l}}^{\beta_{l}}\right)\right), I \circ \mathcal{Q}_{\kappa_{l}}^{0}\right) \geq \bar{\gamma},
$$

for all $l$. Without loss of generality we may assume that $\kappa_{l} \rightarrow \kappa_{0} \in[0,1]$. Since the measures $\mathcal{Q}_{\kappa}^{0}, \kappa \in(0,1]$, were obtained as the limit (2.23), then by (1.5) and the Fatou lemma we have

$$
\int\|\psi\|_{2}^{2 p} \mathcal{Q}_{\kappa}^{0}(d \psi) \leq C_{p}^{*}
$$

for each $p$ and each $\kappa$. The block-structure of the effective equation and this estimate immediately imply that $\mathcal{Q}_{\kappa}^{0}$ continuously depends on $\kappa$ in the space of measures on $X^{2-\gamma}$. From this continuity and (2.29) we get that

$$
\operatorname{dist}\left(\mathcal{D}\left(I\left(v_{\kappa_{l}}^{\beta_{l}}\right)\right), I \circ \mathcal{Q}_{\kappa_{0}}^{0}\right) \geq \frac{1}{2} \bar{\gamma} \quad \forall l \geq \bar{\ell},
$$

for a suitable $\bar{\ell}$.

Now consider the sequence $\left\{a_{\kappa_{l}}^{\beta_{l}}(t)\right\}$, where $a_{\kappa_{l}}^{\beta_{l}}(t)$ is a solution of $(2.10)$ with $\beta=\beta_{l}$ and $\kappa=\kappa_{l}$. Literally repeating the first part of the theorem's proof we see that, replacing the sequence $\{l \rightarrow \infty\}$ by a suitable subsequence $\left\{l^{\prime} \rightarrow \infty\right\}$, we have the weak convergence in $X^{2-\gamma}$

$$
\mathcal{D}\left(a_{\kappa_{l^{\prime}}}^{\left.\beta_{l^{\prime}}(\cdot)\right)} \rightarrow \mathcal{Q}_{\kappa_{0}}^{0} \quad \text { as } \quad l^{\prime} \rightarrow \infty\right.
$$

in contradiction with (2.30).

\subsection{Averaging for stationary solutions.}

Our presentation in this section is sketchy since the argument is similar to that in the previous section, and missing details can be found in [KM13].

Let $\mu^{\beta}$ be the stationary measure for eq. (2.1), which is unique by Theorem 1.1, and $\tilde{v}^{\beta}(t), 0 \leq t<\infty$, be a corresponding stationary solution. Let $\bar{\mu}^{\beta}=\left.\mathcal{D}\left(\tilde{v}^{\beta}\right)\right|_{0 \leq t<\infty}$. Consider the actions $I\left(\tilde{v}^{\beta}(t)\right)$ as in (0.12). Since $\tilde{v}^{\beta}$ inherits the a-priori estimates (1.3) and (1.5), 
then a stationary analogy of the convergence (2.24) holds. Namely, for any $\gamma>0$ there exists a measure $\mathcal{Q}$ on $C\left([0, \infty), h_{I}^{2-\gamma}\right)=: \bar{X}_{I}^{2-\gamma}$ and a sequence $\beta_{l} \rightarrow \infty$ such that

$$
\mathcal{D}\left(I\left(\tilde{v}^{\beta_{l}}(\cdot)\right)\right) \rightarrow \mathcal{Q} \quad \text { as } \beta_{l} \rightarrow \infty,
$$

weakly in $\bar{X}_{I}^{2-\gamma}$. The measure $\mathcal{Q}$ is stationary with respect to translations of $t$.

Replacing the sequence $\left\{\beta_{l}\right\}$ by a suitable subsequence we achieve that the stationary measures $\mu^{\beta_{l}}=\left.\bar{\mu}^{\beta_{l}}\right|_{t=\text { const }}$ converge, weakly in $h^{2-\gamma}$, to some measure $m^{0}$. Clearly, $I \circ m^{0}$ is the marginal distribution for $\mathcal{Q}$ as $t=$ const.

Consider a solution $v^{0}(t)$ of the effective equation such that $\mathcal{D}\left(v^{0}(0)\right)=m^{0}$, and compare it with $a^{\beta}(t)$ which is the solution $\tilde{v}^{\beta}(t)$, written in the interaction representation. Then $\mathcal{D}\left(I\left(a^{\beta}(\cdot)\right)=\mathcal{D}\left(I\left(\tilde{v}^{\beta}(\cdot)\right)\right.\right.$. Since $\mathcal{D}\left(a^{\beta_{l}}(0)\right) \rightarrow \mathcal{D}\left(v^{0}(0)\right)$, then for the same reason as in Section $2.3, \mathcal{D}\left(a^{\beta_{l}}(\cdot)\right) \rightarrow \mathcal{D}\left(v^{0}(\cdot)\right)$, if we replace the sequence $\left\{\beta_{l}\right\}$ by a subsequence. So

$$
\mathcal{D}\left(I\left(a^{\beta_{l}}(\cdot)\right)\right)=\mathcal{D}\left(I\left(\tilde{v}^{\beta_{l}}(\cdot)\right)\right) \rightarrow \mathcal{D}\left(I\left(v^{0}(\cdot)\right),\right.
$$

and $I \circ \mathcal{D}\left(v^{0}(\cdot)\right)=\mathcal{Q}$ by $(2.31)$. Therefore, $I \circ \mathcal{D}\left(v^{0}(t)\right)=I \circ m^{0}$ for any $t$. Taking the limit as $t \rightarrow \infty$ using Lemma 2.3 we get that $I \circ \mu^{0}=I \circ m^{0}$, where $\mu^{0}$ is the unique stationary measure for the effective equation. That is,

$$
I \circ \mu^{0}=\lim _{\beta_{l} \rightarrow \infty} I \circ \mu^{\beta_{l}} .
$$

Since the stationary measure $\mu^{0}$ is unique, this convergence holds as $\beta \rightarrow \infty$. For the same reason as in Section 2.3, it is uniform in $\kappa \in(0,1]$, and we have

Theorem 2.8. If $\mu^{\beta}$ is the unique stationary measure for eq. (2.1) and $\mu^{0}$ is the unique stationary measure for the effective equation (2.6), then $\lim _{\beta \rightarrow \infty} I \circ \mu^{\beta}=I \circ \mu^{0}$, and the convergence is uniform in $\kappa \in(0,1]$. For any solution $v^{\beta}(t)$ of $(1.1)$ with $\beta$-independent initial data $v^{\beta}(0) \in h^{2}$, we have

$$
\lim _{\beta \rightarrow \infty} \lim _{t \rightarrow \infty} \mathcal{D}\left(I\left(v^{\beta}(t)\right)\right)=I \circ \mu^{0} .
$$

\section{Proof of Lemma 2.7}

We restrict ourselves to demonstrating (2.27) since the proof of (2.28) is similar. We adopt a notation from [KP08]. Namely, we denote by $\varkappa(t)$ various functions of $t$ such that $\varkappa \rightarrow 0$ as $t \rightarrow \infty$, and denote by $\varkappa_{\infty}(t)$ functions, satisfying $\varkappa(t)=o\left(t^{-n}\right)$ for each $n$. We write $\varkappa(t ; M)$ to indicate that $\varkappa(t)$ depends on a parameter $M$. Besides for events $Q$ and $O$ and a random variable $f$ we write $\mathbf{P}_{O}(Q)=\mathbf{P}(O \cap Q)$ and $\mathbf{E}_{O}(f)=\mathbf{E}\left(\chi_{O} f\right)$.

The constants below may depend on $\mathbf{k}$, but this dependence is not indicated since $\mathbf{k}$ is fixed through the proof of the lemma. By $M$ and $N$ we denote suitable functions of $\beta$ such that $N \geq\left|\mathbf{k}_{L}\right|$,

$$
M(\beta), N(\beta) \rightarrow \infty \quad \text { as } \beta \rightarrow \infty,
$$

but

$$
\beta^{-1}\left(M^{n}+N^{n}\right) \rightarrow 0 \quad \text { as } \beta \rightarrow \infty, \quad \forall n .
$$

We recall that a notation $v^{N}$, where $N \in \mathbb{N}$ and $v$ is a vector $\left(v_{\mathbf{k}}, \mathbf{k} \in \mathbb{Z}_{*}^{2}\right)$, is defined in Notation. 
Define

$$
\mathfrak{A}_{\mathbf{k}, N}^{\beta}:=\mathbf{E} \max _{0 \leq t \leq T}\left|\int_{0}^{t} \mathcal{R}_{\mathbf{k}}\left(a^{\beta, N}(s), \beta s\right) d s\right| .
$$

As $\mathcal{R}=\mathbf{R}-R$, then by (2.8) and (2.13) the quadratic function $\mathcal{R}_{\mathbf{k}}$ satisfies

$$
\left|\mathcal{R}_{\mathbf{k}}(a, \tau)\right| \leq C|a|_{h^{1}}^{2} \quad \forall \tau .
$$

Since

$$
\left|v-v^{N}\right|_{h^{1}}^{2}=\sum_{\left|\mathbf{n}_{L}\right|>N}\left|\mathbf{n}_{L}\right|^{2}\left|v_{\mathbf{n}}\right|^{2} \leq N^{-2}|v|_{h^{2}}^{2},
$$

then we have

$$
\begin{aligned}
\left|\mathfrak{A}_{\mathbf{k}}^{\beta}-\mathfrak{A}_{\mathbf{k}, N}^{\beta}\right| & \leq \mathbf{E} \int_{0}^{T}\left|\mathcal{R}_{\mathbf{k}}\left(a^{\beta}(s), \beta s\right)-\mathcal{R}_{\mathbf{k}}\left(a^{\beta, N}(s), \beta s\right)\right| d s \\
& \leq C \mathbf{E} \int_{0}^{T}\left|a^{\beta}\right|_{h^{1}}\left|a^{\beta}-a^{\beta, N}\right|_{h^{1}} d s \\
& \leq \frac{C}{N} \mathbf{E} \int_{0}^{T}\left|a^{\beta}\right|_{h^{2}}^{2} d s \leq \varkappa(N) .
\end{aligned}
$$

Denote by $\Omega_{M}=\Omega_{M}^{\beta}$ the event

$$
\Omega_{M}=\left\{\sup _{0 \leq \tau \leq T}\left|a^{\beta}(\tau)\right|_{h^{2}} \leq M\right\} .
$$

Then, by (1.5), $\mathbf{P}\left(\Omega_{M}^{c}\right) \leq \varkappa_{\infty}(M)$, and using (3.2) we get

$$
\begin{aligned}
\mathbf{E}_{\Omega_{M}^{c}} \mathfrak{A}_{\mathbf{k}, N}^{\beta} & \leq \int_{0}^{T} \mathbf{E}_{\Omega_{M}^{c}}\left|\mathcal{R}_{\mathbf{k}}\left(a^{\beta, N}(s), \beta s\right)\right| d s \\
& \leq C\left(\mathbf{P}\left(\Omega_{M}^{c}\right)\right)^{1 / 2} \int_{0}^{T}\left(\mathbf{E}\left|a^{\beta}\right|_{h^{1}}^{4}\right)^{1 / 2} d s \leq \varkappa_{\infty}(M) .
\end{aligned}
$$

So $\mathfrak{A}_{\mathbf{k}, N}^{\beta} \leq \varkappa_{\infty}(M)+\mathfrak{A}_{\mathbf{k}, N, M}^{\beta}$, where

$$
\mathfrak{A}_{\mathbf{k}, N, M}^{\beta}:=\mathbf{E}_{\Omega_{M}} \max _{0 \leq t \leq T}\left|\int_{0}^{t} \mathcal{R}_{\mathbf{k}}\left(a^{\beta, N}(s), \beta s\right) d s\right| .
$$

Consider a partition of $[0, T]$ by the points

$$
\tau_{n}=n L, \quad 0 \leq n \leq K \sim T / L, \quad L=\beta^{-1 / 2} .
$$

where $\tau_{K}$ is the last point $\tau_{n}$ in $[0, T)$. Let us denote

$$
\eta_{l}=\int_{\tau_{l}}^{\tau_{l+1}} \mathcal{R}_{\mathbf{k}}\left(a^{\beta, N}(s), \beta s\right) d s, \quad 0 \leq l \leq K-1 .
$$


Since for $\omega \in \Omega_{M}$ and any $\tau^{\prime}<\tau^{\prime \prime}$ such that $\tau^{\prime \prime}-\tau^{\prime} \leq L$ in view of $(3.2)$ we have

$$
\left|\int_{\tau^{\prime}}^{\tau^{\prime \prime}} \mathcal{R}_{\mathbf{k}}\left(a^{\beta, N}(s), \beta s\right) d s\right| \leq L C(M),
$$

then

$$
\mathfrak{A}_{\mathbf{k}, N, M}^{\beta} \leq L C(M)+\mathbf{E}_{\Omega_{M}} \sum_{l=0}^{K-1}\left|\eta_{l}\right| .
$$

Fix any $r<-1$ and consider the event

$$
\mathcal{F}_{l}=\left\{\sup _{\tau_{l} \leq s \leq \tau_{l+1}}\left|a^{\beta}(s)-a^{\beta}\left(\tau_{l}\right)\right|_{h^{r}} \geq L^{1 / 4}\right\} .
$$

By the equicontinuity of the processes $\left\{a^{\beta}(t)\right\}$ on suitable events with arbitrarily close to one $\beta$-independent probability (as shown in Section 2.3), the probability of $\mathbf{P}\left(\mathcal{F}_{l}\right)$ goes to zero with $L$, uniformly in $l$ and $\beta$. Since $\left|\eta_{l}\right| \leq C(M) L$ for $\omega \in \Omega_{M}$ and for each $l$, then

$$
\sum_{l=0}^{K-1}\left|\mathbf{E}_{\Omega_{M}}\right| \eta_{l}\left|-\mathbf{E}_{\Omega_{M} \backslash \mathcal{F}_{l}}\right| \eta_{l}|| \leq C(M) L \sum_{l=0}^{K-1} \mathbf{P}_{\Omega_{M}}\left(\mathcal{F}_{l}\right) \leq C(M) \varkappa\left(L^{-1}\right),
$$

and it remains to estimate $\sum_{l} \mathbf{E}_{\Omega_{M} \backslash \mathcal{F}_{l}}\left|\eta_{l}\right|$.

We have

$$
\begin{aligned}
\left|\eta_{l}\right| & \leq\left|\int_{\tau_{l}}^{\tau_{l+1}}\left(\mathcal{R}_{\mathbf{k}}\left(a^{\beta, N}(s), \beta s\right)-\mathcal{R}_{\mathbf{k}}\left(a^{\beta, N}\left(\tau_{l}\right), \beta s\right)\right) d s\right| \\
& +\left|\int_{\tau_{l}}^{\tau_{l+1}}\left(\mathcal{R}_{\mathbf{k}}\left(a^{\beta, N}\left(\tau_{l}\right), \beta s\right)\right) d s\right|=: \Upsilon_{l}^{1}+\Upsilon_{l}^{2} .
\end{aligned}
$$

By (3.2), in $\Omega_{M}$ the following inequalities hold:

$$
\begin{aligned}
\left|\mathcal{R}_{\mathbf{k}}\left(a^{\beta, N}(s), \beta s\right)-\mathcal{R}_{\mathbf{k}}\left(a^{\beta, N}\left(\tau_{l}\right), \beta s\right)\right| & \leq C M\left|a^{\beta, N}(s)-a^{\beta, N}\left(\tau_{l}\right)\right|_{h^{1}} \\
& \leq C M N^{1-r}\left|a^{\beta, N}(s)-a^{\beta, N}\left(\tau_{l}\right)\right|_{h^{r}} .
\end{aligned}
$$

So that, by the definition of $\mathcal{F}_{l}$,

$$
\sum_{l} \mathbf{E}_{\Omega_{M} \backslash \mathcal{F}_{l}} \Upsilon_{l}^{1} \leq L^{1 / 4} C(N, M)=\varkappa\left(\beta^{1 / 8} ; N, M\right) .
$$

It remains to estimate the expectation of $\sum \Upsilon_{l}^{2}$. Abbreviating $a^{\beta}$ to $a$ and writing $s \in$ $\left[\tau_{l}, \tau_{l+1}\right]$ as $s=\tau_{l}+\tau, 0 \leq \tau \leq L$, we write $\mathcal{R}_{\mathbf{k}}\left(a^{N}\left(\tau_{l}\right), \beta s\right)$ as

$$
\frac{\rho}{L\left(K+\left|\mathbf{k}_{L}\right|^{2}\right)} \sum_{\substack{\mathbf{j}_{L}|\leq N,| \mathbf{n}_{L} \mid \leq N \\ \lambda_{\mathbf{j}}+\lambda_{\mathbf{n}}-\lambda_{\mathbf{k}} \neq 0}}\left|\mathbf{n}_{L}\right|^{2}(\mathbf{j} \times \mathbf{n}) a_{\mathbf{j}}\left(\tau_{l}\right) a_{\mathbf{n}}\left(\tau_{l}\right) \delta_{\mathbf{k}}^{\mathbf{j n}} e^{-i \beta \tau_{l}\left(\lambda_{\mathbf{j}}+\lambda_{\mathbf{n}}-\lambda_{\mathbf{k}}\right)} e^{-i \beta \tau\left(\lambda_{\mathbf{j}}+\lambda_{\mathbf{n}}-\lambda_{\mathbf{k}}\right)} .
$$


Now consider the torus $\mathbb{T}^{\tilde{N}}=\left\{\varphi_{\mathbf{k}}:\left|\mathbf{k}_{L}\right| \leq N\right\}$, and the trigonometrical polynomial of degree three, defined on $\mathbb{T}^{\tilde{N}}$ :

$$
\begin{aligned}
f_{l}(\varphi)=\frac{\rho}{L\left(K+\left|\mathbf{k}_{L}\right|^{2}\right)} \sum_{\substack{\mathbf{j}_{L}|\leq N,| \mathbf{n}_{L} \mid \leq N \\
\lambda_{\mathbf{j}}+\lambda_{\mathbf{n}}-\lambda_{\mathbf{k}} \neq 0}}\left(\left|\mathbf{n}_{L}\right|^{2}(\mathbf{j} \times \mathbf{n}) a_{\mathbf{j}}\left(\tau_{l}\right) a_{\mathbf{n}}\left(\tau_{l}\right)\right. \\
\left.\times \delta_{\mathbf{k}}^{\mathbf{j n}} e^{-i \beta \tau_{l}\left(\lambda_{\mathbf{j}}+\lambda_{\mathbf{n}}-\lambda_{\mathbf{k}}\right)} e^{-i\left(\varphi_{\mathbf{j}}+\varphi_{\mathbf{n}}-\varphi_{\mathbf{k}}\right)}\right) .
\end{aligned}
$$

Its coefficients are bounded by a constant $C(M, N)$, and $\mathcal{R}_{\mathbf{k}}\left(a^{N}\left(\tau_{l}\right), \beta s\right)=f_{l}\left(\beta \tau \Lambda^{N}\right)$.

We have

$$
\Upsilon_{l}^{2}=\left|\int_{\tau_{l}}^{\tau_{l+1}}\left(\mathcal{R}_{\mathbf{k}}\left(a^{\beta, N}\left(\tau_{l}\right), \beta s\right)\right) d s\right|=\left|\int_{0}^{L} f_{l}\left(\beta \tau \Lambda^{N}\right) d \tau\right|=\beta^{-1}\left|\int_{0}^{\beta L} f_{l}\left(t \Lambda^{N}\right) d t\right| .
$$

Since in the sum, which defines $f_{l}$, the indexes $\mathbf{j}$ and $\mathbf{n}$ are such that $\lambda_{\mathbf{j}}+\lambda_{\mathbf{n}}-\lambda_{\mathbf{k}} \neq 0$, then in view of (1.14), $\left\langle f_{l}\right\rangle_{\Lambda^{N}}=0$. Accordingly, by Lemma 1.6,

$$
\Upsilon_{l}^{2} \leq L \varkappa(\beta L ; N, M, \Lambda) .
$$

Therefore

$$
\sum_{l} \mathbf{E}_{\Omega_{M} \backslash \mathcal{F}_{l}} \Upsilon_{l}^{2} \leq \varkappa\left(\beta^{1 / 2} ; N, M, \Lambda\right)
$$

Now the inequalities (3.3)-(3.7) imply that

$$
\mathfrak{A}_{\mathbf{k}}^{\beta} \leq \varkappa(N)+\varkappa_{\infty}(M)+\varkappa(\beta ; M)+\varkappa(\beta ; N, M)+\varkappa(\beta ; N, M, \Lambda) .
$$

Choosing first $N$ and $M$ large, and then $\beta$ large, in such a way that (3.1) holds, we make the r.h.s. arbitrarily small. This proves the lemma.

\section{A The limit of large box.}

Let us consider eq. (0.2), where the boundary condition $\mathbf{x} \in \mathbb{T}_{L, 1}^{2}$ is replaced by $\mathbf{x} \in \mathbb{T}_{\theta}^{2}$, see (0.13). Now the eigenfunctions of the operator of Rossby waves $(-\Delta+K)^{-1} \circ \frac{\partial}{\partial x}$ are $e^{i\left(x k_{x} L^{-1}+y k_{y}\right)}$, where $\mathbf{k}=\left(k_{x}, k_{y}\right) \in \theta^{-1} \mathbb{Z}^{2}=: \mathbb{Z}_{\theta *}^{2}$, and the corresponding frequencies of the Rossby waves are

$$
\lambda_{\mathbf{k}}=\lambda_{\mathbf{k}}^{\theta, L, K}=\frac{k_{x} L}{L^{2} K+k_{x}^{2}+L^{2} k_{y}^{2}}, \quad \mathbf{k} \in \mathbb{Z}_{\theta *}^{2} .
$$

For the same reason as in Section 1.2, for any $K \geq 0$ and $\theta>0$ the set of positive $L$ such that the spectrum $\left\{\lambda_{\mathbf{k}}\right\}$ is strongly resonant, is at most countable (if $K=0$, then this set does not depend on $\theta$ ).

Repeating the argument of Section 2 we see that for any $L$ outside that countable set, the resonant part $R$ of the nonlinearity $P$ in eq. (2.1) is given by (2.4), where now the sum is taken over indexes $\mathbf{j}, \mathbf{n} \in \mathbb{Z}_{\theta *}^{2}$, and that the resonant hamiltonian equation (2.14) still has the form (2.16), (2.15), where $\mathbf{k} \in \mathbb{Z}_{\theta *}^{2}$. The assertions of Theorems 2.6, 2.8 remain true for any $\theta>0$. 
When $\theta$ grows to infinity, the lattice $\mathbb{Z}_{\theta *}^{2}$ becomes dense in $\mathbb{R}^{2}$, and relation (2.6) defines a field of nonlinear stochastic rotators on this lattice. Denote $J_{\mathbf{k}}=\frac{1}{2}\left(\left|v_{\mathbf{k}}\right|^{2}+\left|v_{\overline{\mathbf{k}}}\right|^{2}\right)$. Since this is an integral of motion for the hamiltonian system (2.16), then applying the Ito formula to a stationary solution $v(t)$ of $(2.6)$ we get that $2 \gamma_{\mathbf{k}} \mathbf{E} J_{\mathbf{k}}=b_{\mathbf{k}}^{2}+b_{\overline{\mathbf{k}}}^{2}$. So $\mathbf{E} \frac{1}{2}\left(\left|v_{\mathbf{k}}\right|^{2}+\left|v_{\overline{\mathbf{k}}}\right|^{2}\right)=\frac{b_{\mathbf{k}}^{2}+b_{\mathbf{k}}^{2}}{2 \gamma_{\mathbf{k}}}$, and when $\theta \rightarrow \infty$ the energy spectrum of the stationary solution is concentrated where the spectrum of the random force is. That is, under the double limit $\beta \rightarrow \infty$ and then $\theta \rightarrow \infty$ the equation (0.2) also does not exhibit the energy transfer to high modes.

\section{References}

[AJW11] Mustafa A. H. Al-Jaboori and Djoko Wirosoetisno, Navier-Stokes equations on the $\beta$-plane, Discrete Contin. Dyn. Syst. Ser. B 16 (2011), no. 3, 687-701.

[BDW98] J. R. Brannan, J. Duan, and T. Wanner, Dissipative quasi-geostrophic dynamics under random forcing, J. Math. Analysis Appl. 228 (1998), 221-233.

[Ber00] D. Bernard, Influence of friction on the direct cascade of the 2d forced turbulence, Europhys. Lett. 50 (2000), 333-339.

[BMN97] A. Babin, A. Mahalov, and B. Nicolaenko, Regularity and integrability of 3D Euler and Navier-Stokes equations for rotating fluids, Asymptot. Anal. 15 (1997), no. 2, $103-150$.

[BMN99]__ Global regularity of 3D rotating Navier-Stokes equations for resonant domains, Indiana Univ. Math. J. 48 (1999), no. 3, 1133-1176.

[BN90] A. M. Balk and S. V. Nazarenko, Physical realizability of anisotropic weak-turbulence Kolmogorov spectra, Sov. Phys. JETP 70 (1990), no. 6, 1031-1041.

[Bou71] N. Bourbaki, Eléments de Mathématique. Livre III: Topologie Génerale, Chap. 5 à 10, Hermann, Paris, 1971.

[CDGG06] J.-Y. Chemin, B. Desjardins, I. Gallagher, and E. Grenier, Mathematical geophysics, The Clarendon Press, Oxford University Press, Oxford, 2006.

[CFT13] C. Cao, A. Farhat, and E. S. Titi, Global well-posedness of an inviscid threedimensional pseudo-Hasegawa-Mima model, Comm. Math. Phys. 319 (2013), no. 1, $195-229$.

[CZ00] P. Caillol and V. Zeitlin, Kinetic equations and stationary energy spectra of weakly nonlinear internal gravity waves, Dynamics of Atmospheres and Oceans 32 (2000), 81-112.

[DG04] S. Danilov and V. M. Gryanik, Barotropic beta-plane turbulence in a regime with strong zonal jets revisited, Journal of the Atmospheric Sciences 61 (2004), 22832295 .

[DZ96] G. Da Prato and J. Zabchyk, Ergodicity for Infinite Dimensional Systems, Cambridge University Press, Cambridge, 1996. 
[FMVE05] C. Franzke, A. Majda, and E. Vanden-Eijnden, Low-order stochastic mode reduction for a realistic barotropic model climate, Journal of the Atmospheric Sciences 62 (2005), 1722-1745.

[FW84] M. Freidlin and A. Wentzell, Random Perturbations of Dynamical Systems, Springer-Verlag, New York, 1984.

[GSR06] I. Gallagher and L. Saint-Raymond, Mathematical study of the betaplane model: equatorial waves and convergence results, Mém. Soc. Math. Fr. (N.S.) (2006), no. $107, \mathrm{v}+116$ pp. (2007).

[Kar94] E. Kartashova, Weakly nonlinear theory of finite-size effects in resonators, Phys. Rev. Lett. 72 (1994), 2013-2016.

[Kar10] _ Nonlinear Resonance Analysis, Cambridge University Press, Cambridge, 2010.

[Kha68] R. Khasminski, On the avaraging principle for Ito stochastic differential equations, Kybernetika 4 (1968), 260-279, (in Russian).

[KM13] S. Kuksin and A. Maiocchi, Resonant averaging for small solutions of stochastic NLS equations, arXiv:1311.6793, 2013.

[KM14] _ Derivation of the Kolmogorov-Zakharov equation from the resonantaveraged stochastic NLS equation, Physica D (2014), to appear, arXiv:1311.6794.

[KP08] S. B. Kuksin and A. L. Piatnitski, Khasminskii - Whitham averaging for randomly perturbed KdV equation, J. Math. Pures Appl. 89 (2008), 400-428.

[KS91] I. Karatzas and S. Shreve, Brownian Motion and Stochastic Calculus, 2nd ed., Springer-Verlag, Berlin, 1991.

[KS12] S. Kuksin and A. Shirikyan, Mathematics of Two-Dimensional Turbulence, Cambridge University Press, Cambridge, 2012.

[Kuk10] S. B. Kuksin, Damped-driven KdV and effective equations for long-time behaviour of its solutions, GAFA 20 (2010), 1431-1463.

[Kuk13] _ Weakly nonlinear stochastic CGL equations, Ann. Inst. H. Poincaré - PR 49 (2013), 1033-1056.

[Maj03] A. Majda, Introduction to PDEs and waves for the atmosphere and ocean, Courant Lecture Notes in Mathematics, vol. 9, New York University, Courant Institute of Mathematical Sciences, New York; American Mathematical Society, Providence, RI, 2003.

[MR99] R. Mikulevicius and B. L. Rozovskii, Martingale problems for stochastic PDEs, Stochastic partial differential equations: six perspectives, Math. Surveys Monogr., no. 64, Amer. Math. Soc., Providence, RI, 1999, pp. 243-325.

[Naz11] S. Nazarenko, Wave Turbulence, Springer, Berlin, 2011. 
[Ped79] J. Pedlosky, Geophysical fluid dynamics, Springer-Verlag, New York, 1979.

[Pit98] L.I. Piterbarg, Hamiltonian formalism for Rossby waves, Amer. Math. Soc. Transl. Ser. 2182 (1998), 131-166.

$\left[\mathrm{R}^{+} 39\right] \quad$ C. G. Rossby et al., Relation between variation in the intensity of the zonal circulation of the atmosphere and the displacements of the semi-permanents centers of action, J. Marine Res. 2 (1939), 38-55.

[RPK93] G. M. Reznik, L. I. Piterbarg, and E. A. Kartashova, Nonlinear interactions of spherical Rossby waves, Dynamics of Atmosphere and Oceans 18 (1993), 235-252.

[Ver97] A. Yu. Veretennikov, On polynomial mixing bounds for stochastic differential equations, Stochastic Processes and their Applications 70 (1997), 115-127.

[Yor74] M. Yor, Existence et unicité de diffusion à valeurs dans un espace de Hilbert, Ann. Inst. Henri Poincaré Sec. B, 10 (1974), 55-88. 